Article original

\title{
L’hypersensibilité à l'électricité : une approche multidisciplinaire pour un problème multifactoriel. Revue de la littérature
}

\author{
M. Crasson \\ Université de Liège, Belgian Bioelectromagnetic Group, Psychoneuroendocrinology Unit, 35, CHU, 4000 Liège, Belgique
}

Reçu le 10 septembre 2003 ; accepté le 13 octobre 2004

\begin{abstract}
Résumé
L'hypersensibilité à l'électricité (HE) est un syndrome essentiellement défini par le patient lui-même, lequel présente des plaintes qu'il attribue à l'usage ou à la proximité d'appareils ou d'équipements émettant des champs électriques (CE), magnétiques (CM) ou électromagnétiques (CEM). La plupart des recherches, scandinaves, indiquent des plaintes dermatologiques chez des travailleurs sur écran mais de plus en plus de personnes se plaignent d'un syndrome plus général de type neurasthénique qui s'étend à d'autres sources de rayonnements nonionisants, lignes à haute tension et systèmes de mobilophonie. Ce terme n'implique pas de relation démontrée entre l'exposition aux champs électromagnétiques et les symptômes rapportés, comme l'indiquent les études de provocation. L'origine de l'hypersensibilité à l'électricité serait davantage multifactorielle et invite à la prise en considération multidisciplinaire de facteurs environnementaux, biologiques, psychologiques et psychosociaux dans le diagnostic et le traitement de ce syndrome hétérogène. Le but de cet article est de donner un aperçu des connaissances actuelles et des hypothèses étiologiques destinées à mieux comprendre et mieux aider les personnes qui en souffrent.

(C) 2005 Elsevier SAS. Tous droits réservés.
\end{abstract}

\begin{abstract}
Hypersensitivity to electricity (EHS) is a self-defined syndrome where individuals experience adverse effects while using or being in the vicinity of devices or equipments emitting electric, magnetic or electromagnetic fields. Most of studies are Scandinavian and indicate dermatological complaints from visual display units workers but more and more individuals complaint about a general syndrome, neurasthenic like, which extend to other sources of non-ionising radiation; power-lines and mobile systems. The use of this term cannot be considered to imply an already established causal relationship between electromagnetic fields and reported symptoms, as shown by provocation studies. The origin of hypersensitivity to electricity appears to be mutifactorial and request a multidisciplinary approach of environmental, biologic, psychologic and psychosocial factors in the diagnostic and treatment of this heterogeneous syndrome. The goal of this paper is to review current knowledge and hypothesis related to this syndrome to better understand and help people suffering from it.
\end{abstract}

(c) 2005 Elsevier SAS. Tous droits réservés.

Mots clés : Hypersensibilité ; Électricité ; Multifactoriel ; Maladie environnementale ; Stress

Keywords: Hypersensitivity; Electricity; Multifactorial; Environmental illness; Stress

\section{Introduction}

Depuis quelques décennies, notre environnement électromagnétique s'est profondément modifié de par l'utilisation croissante de l'électricité dans nos pays industrialisés. Aux sources naturelles, terrestres et cosmiques, s'ajoutent les sources artificielles dues aux nombreux progrès techniques qui

Adresse e-mail : mcrasson@ulg.ac.be (M. Crasson). font usages de l'électricité. La prolifération des lignes de transmission et de distribution de l'électricité, l'utilisation croissante des techniques liées à l'électricité, que ce soit dans le domaine de l'industrie, la médecine, les télécommunications, la recherche, l'éducation et foyers domestiques est à l'origine de champs électromagnétiques qui couvrent tout le spectre des fréquences. Elle ne s'est pas réalisée sans poser certaines questions quant aux risques que pouvaient comporter ces champs électromagnétiques sur la santé des personnes 
et de vastes programmes de recherche ont été rapidement mis en marche dans de nombreux pays du globe depuis une trentaine d'années. Parallèlement à la problématique des cancers, d'autres aspects de la santé et du bien-être ont fait l'objet d'études, réalisées dans un premier temps dans le milieu professionnel.

Un des points de départ de l'étude des symptômes subjectifs liés aux champs électriques et magnétiques à la fréquence du réseau (50 Hz en Europe, $60 \mathrm{~Hz}$ aux États-Unis) se situe en ex-URSS. Des enquêtes médicales réalisées chez des ouvriers occupés à la maintenance de lignes hautetension indiquent une série de symptômes subjectifs non spécifiques tels que céphalées, irritabilité, troubles de la concentration, troubles du sommeil, fatigue, faiblesse musculaire, nausées... Ces symptômes n'ont pas été retrouvés dans d'autres études réalisées dans d'autres compagnies électriques, en Europe, aux États-Unis ou au Canada (revue in Crasson, 2003). Des études épidémiologiques ont été entreprises afin d'évaluer l'incidence de la dépression et du suicide en relation avec les champs électriques et magnétiques $50 \mathrm{~Hz}$ (études résidentielles et professionnelles), avec des résultats variés. Quant aux études réalisées en laboratoire sur des volontaires en bonne santé, elles n'indiquent généralement pas d'effet de l'exposition contrôlée à des champs magnétiques 50-60 Hz sur l'humeur et le bien-être (revue in International Agency for Research on Cancer (IARC) Monographs, 2002). Les études sur les effets cognitifs de l'exposition aux champs électriques et magnétiques à la fréquence du réseau apparaissent controversés (revue in Crasson, 2003). Elles indiquent tantôt une amélioration des performances, tantôt une détérioration et tantôt une absence d'effet. Les résultats sont par ailleurs difficilement reproductibles.

Dans la gamme des radiofréquences, des études ont été réalisées sur la prévalence de symptômes subjectifs chez des utilisateurs de téléphones mobiles. Globalement, ces études indiquent une augmentation des symptômes subjectifs (dont des sensations de chaleur au niveau de l'oreille ou de la face, céphalées, inconfort, vertiges, difficultés de concentration, fatigue) avec l'augmentation du temps d'appel et du nombre d'appels par jour (Al-Khaiwi et Meo, 2004 ; Chia, et al., 2000 ; Hocking, 1998 ; Oftedal et al., 2000 ; Sandström et al., 2001 ; Santini et al., 2001a ; Wilen et al., 2003). Ces symptômes ont été mis en relation avec les taux d'absorption spécifique (Wilen et al., 2003) et expliqués hypothétiquement par une stimulation vestibulaire (Cox et Luxton, 2000). Des différences sont parfois observées en fonction du type de téléphone mobile utilisé $(900 \mathrm{MHz}, 1800 \mathrm{MHz}$, numérique vs analogique). Une étude a été réalisée en France chez des personnes habitant à proximité de station de base de téléphonie mobile (jusqu'à 300 m) (Santini et al., 2001b). Les réponses de ces personnes ont été comparées à celles des personnes résidant à plus de $300 \mathrm{~m}$ d'une antenne. Les résultats indiquent des plaintes plus fréquentes lorsqu'on se rapproche des stations de base et différenciées en fonction de la distance. Ce type d'étude présente de nombreux biais dont celui de demander aux habitants de préciser eux-mêmes leur éloignement par rapport à la station relais, la relation ostensible dans cette enquête, pour les personnes interrogées, entre leurs symptômes et l'antenne ainsi que l'absence de mesure des champs. Cette étude ne permet pas de conclure à une relation avec les champs émis. Enfin, une étude a été réalisée en laboratoire, en double insu, sur des volontaires en bonne santé et indique qu'une exposition à des radiofréquences (RF) $(902 \mathrm{MHz}$, modulation à $217 \mathrm{~Hz}$ ) de 30 ou 60 minutes n'entraîne pas de symptômes subjectifs chez des personnes en bonne santé (Koivisto et al., 2001). Les effets cognitifs ont également fait l'objet d'études. Leurs conclusions sont similaires à celles obtenues dans les études de la gamme 50-60 Hz. Les résultats obtenus ne permettent pas de conclure à la présence ou l'absence d'effets reproductibles sur les performances cognitives (revue in D'Andrea et al., 2003).

Les études réalisées dans la population générale sont généralement trop peu nombreuses ou ne permettent pas de conclure que les champs électromagnétiques auxquels nous sommes exposés dans notre vie quotidienne altèrent le bienêtre ou détériorent la qualité de vie. Nous constatons aujourd'hui qu'un nombre croissant de personnes se plaignent d'une large gamme de symptômes non spécifiques, qu'elles attribuent à l'électricité et qui apparaissent pour des niveaux d'exposition qui ne causent aucune réaction chez la majorité des autres personnes, des niveaux de fréquence et d'intensité auxquels la population générale est exposée et largement en dessous des recommandations internationales. Ces personnes se disent hypersensibles à l'électricité et cette appellation pose certaines questions qui se réfèrent à l'établissement de critères diagnostiques, à sa prévalence, à la recherche d'une étiologie ainsi que du traitement le plus adapté.

\section{Un problème de diagnostic}

Il n'existe pas de définition formelle ou de diagnostic d'HE en raison de la non-spécificité des symptômes rapportés, de l'apparente hétérogénéité des personnes affligées et de l'absence d'étiologie établie. Seule une définition opérationnelle existe à l'heure actuelle. L'hypersensibilité à l'électricité est un syndrome essentiellement défini par le patient luimême, lequel présente des plaintes qu'il attribue à l'usage ou à la proximité d'appareils ou d'équipements émettant des champs électriques (CE), magnétiques (CM) ou électromagnétiques (CEM) (Bergqvist et Vogel, 1997). La recherche de symptômes associés à l'électricité a débuté en Norvège et en Suède au début des années 1980 à partir de plaintes dermatologiques exprimées par des travailleurs sur écran. Aujourd'hui, un nombre croissant de personnes se plaignent d'être hypersensibles à l'électricité. Les symptômes varient d'un individu à l'autre mais dans la majorité des cas, ils sont non spécifiques, de faible ampleur et sans signes objectifs. Il existe cependant des cas où des personnes présentent des problèmes de santé plus sévères ou une altération plus importante de leur qualité de vie. Les sources de champs associées à ces symptômes peuvent être les écrans d'ordinateur, des 
appareils électriques domestiques, les lignes à haute tension, les lampes fluorescentes, les téléphones mobiles et/ou les antennes-relais ou encore le travail sur radar ou autre équipement produisant des radiofréquences et cette liste n'est pas exhaustive. Les symptômes décroissent lorsque ces patients s'éloignent de la source, par exemple lorsqu'ils ne travaillent pas (week-ends, congés...) ou lorsqu'ils sont en vacances. Cependant, des réactions retardées ou prolongées après l'exposition peuvent se produire.

En l'absence de critères diagnostics, les symptômes observés sont attribués à ce que Knave, et al., (1992), vont intituler « hypersensibilité à l'électricité », expression qui trouvera par la suite d'autres synonymes tels que «électrosensibilité » (electrosensitivity), « hypersensibilité électromagnétique » (electromagnetic hypersensitivity), « hypersensibilité électrique » (electrical hypersensitivity), « sensibilité électrique » (electrical sensitivity) ou encore hypersensibilité aux champs électriques et magnétiques » (hypersensitivity to electric and magnetic fields) lorsque celle-ci est restreinte aux champs de basse fréquence (Levallois, 2002). Si le terme « allergie à l'électricité » est parfois cité, il est préférable de ne pas l'utiliser car il ne s'agit pas, au vu des connaissances actuelles, d'une pathologie qui donne lieu à des réactions de type allergique.

Ces patients ne forment pas un groupe homogène en termes de niveau scolaire, de statut socioéconomique ou encore en fonction des symptômes rapportés. Les symptômes sont dermatologiques (picotements, sensations de chaleur ou de brûlures, démangeaisons, rougeurs) et/ou neurologiques (céphalées, troubles de la concentration, vertiges, fatigue, troubles de la concentration, problèmes mnésiques...), mais on retrouve également des symptômes gastro-intestinaux, oculaires, cardiovasculaires, etc., sans qu'aucune lésion organique ne puisse être mise en évidence. D'aucuns insistent sur la distinction entre deux sous-groupes de patients hypersensibles (Berg, 1988 ; Bergdahl, 1995 ; Bergqvist et Vogel, 1997 ; Eriksson et al., 1997a ; Hillert et al., 1999) en fonction de l'attribution du problème. Le premier sous-groupe comprend des patients qui se plaignent de problèmes dermatologiques associés au travail sur ordinateur, les symptômes étant le plus souvent localisés au niveau de la face. Subjectifs (douleurs, sensations de brûlures, démangeaisons) ou objectivables mais non spécifiques (rougeurs, peau sèche ou rosée, eczéma, acné, rosacée, dermatite atopique), les symptômes sont généralement transitoires (réduits le week-end ou après le travail) et de meilleur pronostic (Eriksson et al., 1997a ; Liden, 1996). Il s'agit du « syndrome dermatologique » (Levallois, 2002), aussi appelé « dermatite de l'écran » qui a émergé en Suède et en Norvège au début des années 1980 (Hillert et KolmodinHedman, 1997 ; Bergqvist et Vogel, 1997). Le second sousgroupe se plaint de symptômes associés aux « appareils électriques » en général (Bergdahl, 1995) appelé par Levallois (2002) « syndrome général » et par Stenberg et al. (2002), « hypersensibilité à l'électricité », syndrome d'apparition plus récente et moins bien défini. Le pronostic est relativement moins favorable, dans la mesure où les tentatives de remédia- tion s'avèrent peu efficaces, les comportements d'évitement croissants menant à une incapacité à travailler et un isolement social parfois très important (Hillert et al., 1999, Stenberg, 2002). Cependant, il apparaît souvent que ces deux groupes se chevauchent car leur distinction se fonde à la fois sur l'attribution du problème et sur la nature des symptômes. Il n'est pas rare de constater des symptômes dermatologiques associés aux écrans qui s'étendent à des symptômes de type fatigue, céphalées, troubles de la concentration, etc., ainsi qu'une généralisation de ces symptômes à d'autres sources de champs, d'autres fréquences, intensités et durées d'exposition. Il est important de souligner que le diagnostic repose essentiellement sur les éléments fournis par le patient et sur sa perception de l'attribution causale des symptômes éprouvés. Il n'implique pas nécessairement une relation établie entre l'exposition aux champs électromagnétiques et les réactions sanitaires.

Les plaintes décrites dans les cas d'HE couvrent une série de symptômes non spécifiques également présents dans d'autres syndromes tels que la « sensibilité chimique multiple» («Multiple Chemical Sensitivity» ou MCS), le « Sick Building Syndrome » (SBS), les symptômes liés aux amalgames dentaires, le «Syndrome de Fatigue Chronique » (SFC), la fibromyalgie et le « syndrome de la guerre du Golf » (Kipen et Fiedler, 2002) ou encore « Environmental Somatization Syndrome » (ESS, Göthe, et al., 1995). Seuls les agents responsables attribués à ces symptômes varient. Ensemble, ces ensembles de symptômes récurrents peuvent se regrouper sous le terme de « Intolérances Environnementales Idiopathiques » (« Idiopathic Environmental Illness », IEI) » (IPCS/WHO, 1996), lorsqu'ils sont associés à des facteurs environnementaux tolérés par la plupart des gens et non explicables par d'autres désordres psychiatriques ou médicaux connus (Sparks, 2000a). Ils sont parfois regroupés dans la classe des «symptômes médicalement non expliqués » (« medically unexplained symptoms »), en tant que « troubles somatoformes indifférenciés » (« undifferentiated somatoform disorder ») (Hillert, 2001, $\mathrm{PhD}$ thesis) et peuvent également se regrouper sous l'appellation de syndrome fonctionnel somatique ( « functional somatic syndrome»), tel que suggéré par Wessely et al., (1999). Il peut être intéressant de souligner que l'HE, d'après les données actuelles, ne prédispose et n'est pas un indicateur de pathologie clinique connue (Hillert et al., 1999). L'absence de mécanisme physique et physiologique complique la compréhension de ce syndrome qui acquiert cependant un intérêt grandissant auprès du public et conduit à la création de groupes d'entraide très actifs dans certains pays, notamment en Suède.

\section{Prévalence}

L'occurrence et l'apparition de l'HE varient d'un pays à l'autre, avec davantage de symptômes dermatologiques associés au travail sur ordinateur dans les pays scandinaves et notamment en Suède et davantage de symptômes de type neu- 
rologique (céphalées, fatigue, troubles cognitifs, etc.) associés aux lignes à haute tension ou à la mobilophonie dans nos contrées (Bergqvist et Vogel, 1997, Silny, 1999). Aux ÉtatsUnis et en Grande-Bretagne, les réactions aux champs électromagnétiques sont souvent exprimées chez des personnes qui se plaignent déjà d'allergies chimiques multiples (syndrome chimique multiple ou SCM). D'après l'enquête de la communauté européenne (DGIX) réalisée à partir de questionnaires envoyés à quelques 138 centres de médecine du travail et 15 groupes d'entraide dans 18 pays d'Europe, la prévalence de ce syndrome serait de moins de quelques cas par million (estimation des centres de médecine du travail) à quelques dixièmes de pourcent (estimation des groupes d'entraide) dans les pays nordiques. Les estimations sont plus importantes pour les groupes d'entraide ainsi que dans certains pays comme la Suède, l'Allemagne et le Danemark par comparaison à d'autres pays comme le Royaume-Uni, l'Autriche ou la France. Aucune réponse au questionnaire n'a été fournie par certains pays dont la Belgique ou le Luxembourg, la Grèce, le Portugal ou l'Espagne. Le taux de participation était cependant relativement faible pour les centres de médecine du travail (45\%, $66 \%$ pour les groupes d'entraide). Les groupes d'entraide notent également des problèmes allergiques, des problèmes liés aux amalgames dentaires ou à la sensibilité chimique multiple chez les personnes qui se plaignent d'HE.

La prévalence inégale de ces symptômes en fonction des pays, contrairement à l'utilisation comparable des ordinateurs et autres équipements électriques pourraient s'expliquer par plusieurs facteurs dont une connaissance insuffisante du syndrome, une variation géographique dans la distribution des facteurs contributifs autres que les champs électromagnétiques ou encore par le rôle des médias et de la société (Hillert et Kolmodin-Hedman, 1997). Il est également possible que les problèmes dermatologiques associés au travail sur écran soient considérés comme synonymes d'HE dans certains pays alors qu'ils sont reliés à d'autres facteurs ou appelés différemment dans d'autres pays (Bergqvist et Vogel, 1997).

Une enquête réalisée dans la région de Stockholm dans la population générale (15000 personnes) indique une prévalence de $1,5 \%$, supérieure pour les femmes que pour les hommes et dans les tranches d'âge les plus élevées (60-80 ans). Cette différence de prévalence en fonction de l'âge disparaît cependant lorsque sont retirées les personnes qui souffrent également d'intolérance aux amalgames dentaires. Le groupe de personnes qui répondent positivement à la question : «Are you allergic or hypersensitive to electric or magnetic fields ?» se caractérise également par une prévalence supérieure d'allergies, d'asthme et de rhume des foins, de rhumes et d'hypertension et est plus facilement perturbé par d'autres facteurs environnementaux. Cette enquête indique également que les symptômes sont essentiellement neurovégétatifs, oculaires et respiratoires et que la fatigue est le symptôme le plus souvent rapporté (Hillert et al., 2002a).

Enfin, une enquête téléphonique réalisée auprès de 2072 californiens indique qu'environ $3 \%$ des personnes qui ont participé se qualifient d'hypersensibles aux champs électriques et magnétiques, en répondant positivement à la question : «Are you allergic or very sensitive to getting near electric appliances, computers or power lines ?». Parmi ceux-ci $0,5 \%$ ont dû changer de travail pour cette raison. Parmi les facteurs prédicteurs, les auteurs relèvent un diagnostic médical de SCM, la race/l'ethnie (ni blanc, ni noir, ni hispanique), de faibles revenus et l'incapacité à travailler. Le nombre de personnes qui se plaignent d'HE y est moindre que le nombre de personnes qui se plaignent de SCM (24,4\% de l'échantillon, $n=503$ ), lequel syndrome est caractérisé par un nombre supérieur de femmes et la présence de problèmes asthmatiques. À côté des lignes électriques, les sèchecheveux sont considérés comme la source de CEM la plus fortement associée à leur HE (Levallois et al., 2002). Le Tableau 1 résume les résultats de ces différentes études.

Des études épidémiologiques ont également été réalisées dans les milieux professionnels. Elles ont essentiellement évalué la fréquence des symptômes dermatologiques en relation avec le travail sur écran de visualisation. Elles indiquent des plaintes faciales chez les employés de bureau, plus fréquentes chez les travailleurs sur écran, parfois présentes avec d'autres problèmes de santé tels qu'une irritation oculaire, des symptômes musculosquelettiques et des céphalées. Si les symptômes sont associés à la durée du travail sur écran, elle n'est pas en relation avec le type d'ordinateur (revue in Bergqvist et Vogel, 1997 ; in Levallois, 2002). Les résultats des enquêtes réalisées dans la population générale contrastent quelque peu avec les données récoltées dans les centres de médecine de l'environnement et du travail. Si l'HE concerne toutes les tranches d'âge, la plupart des cas décrits dans ces centres sont des personnes d'âge moyen. Les deux tiers des consultants sont des femmes d'un niveau d'instruction élevé (Bergdahl, 1995 ; Hillert et Kolmodin-Hedman, 1997 ; Hillert et al., 2002a).

Enfin, les données d'une étude suisse, récemment réalisée à partir de questionnaire de santé envoyés à 429 personnes qui se plaignent de symptômes de mauvaise santé attribués à l'exposition aux champs électromagnétiques, indiquent, avec un taux de participation de $92 \%$, des troubles du sommeil dans $58 \%$ des cas, des céphalées dans $41 \%$ des cas, suivis par de la nervosité et de l'angoisse (19\%), de la fatigue (18\%) et des difficultés de concentration dans $16 \%$ des cas. Les symptômes sont le plus souvent mis en relation, dans un ordre décroissant, avec les stations de base de téléphonie, les téléphones mobiles, les téléphones sans fil ou les lignes à hautes tension. La fréquence des dix symptômes les plus cités ne diffère pas en fonction de la source malgré l'apparition de certaines tendances (association écrans et céphalées, techniques de communication et difficultés de concentration, tinnitus, extrêmement basses fréquences, lignes à hautes tension, appareils électriques et nervosité, détresse). Les personnes de cet échantillon sont plus âgées, plus souvent mariées, présentent un niveau scolaire plus élevé et une prévalence de maladie plus élevée que la moyenne de la population suisse (Röösli et al., 2004). 
Tableau 1

Études de prévalence de l'hypersensibilité à l'électricité

\begin{tabular}{|c|c|c|c|c|c|}
\hline Auteurs & Méthode & Population & Prévalence & Facteurs prédicteurs & Particularités \\
\hline $\begin{array}{l}\text { Bergqvist et } \\
\text { Vogel, } 1997 \\
\text { DGV }\end{array}$ & $\begin{array}{l}\text { - Question } \\
\text { - } 1996\end{array}$ & $\begin{array}{l}\text { - Centres de } \\
\text { médecine du } \\
\text { travail }(n=138) \text { et } \\
\text { groupes d'entraide } \\
(n=15) \\
\text { - } 18 \text { pays européens } \\
\text { - TR }=45 \text { et } 66 \%\end{array}$ & $\begin{array}{l}\text { - Suède et } \\
\text { Allemagne } \\
\text { - pas de réponse de } \\
\text { Belgique } \\
\text { - quelques cas par } \\
\text { million à quelques } \\
\text { dizièmes de } \\
\text { pourcent } \\
\text { - } 10 \% \text { des cas sont } \\
\text { sévères }\end{array}$ & $\begin{array}{l}\text { Origine multifactorielle } \\
\text { - facteurs internes } \\
\text { (psychologiques, hormonaux, } \\
\text { dermatologiques...) } \\
\text { - facteurs externes ; physiques (faible } \\
\text { taux d'humidité, lumières } \\
\text { clignotantes) } \\
\text { - facteurs organisationnels (charge de } \\
\text { travail, situation psychosociale) }\end{array}$ & $\begin{array}{l}\text { - symptômes = dermatologiques, du } \\
\text { système nerveux (troubles du } \\
\text { sommeil, troubles de la vigilance, } \\
\text { neurasthénie, stress, irritabilité, } \\
\text { anxiété et céphalées), hormonaux et } \\
\text { métaboliques, etc. } \\
\text { - différences géographiques, } \\
\text { - différences dans l'attribution de la } \\
\text { source }\end{array}$ \\
\hline $\begin{array}{l}\text { Levallois et al. } \\
2002\end{array}$ & $\begin{array}{l}\text { - Enquête- } \\
\text { téléphone } \\
\text { - } 1998\end{array}$ & $\begin{array}{l}\text { - Échantillon } \\
\text { aléatoire, } \\
\text { Californie : } \\
\text { - } n=2072 \\
\text { - TR : } 84 \%\end{array}$ & $\begin{array}{l}\bullet 3,2 \% \mathrm{HE} \\
(n=68 / 2037) \\
\cdot 24,4 \% \mathrm{SCM} \\
(n=503 / 2063) \\
\cdot 1,3 \% \text { HE et pas } \\
\text { SCM }(n=27)\end{array}$ & $\begin{array}{l}\text { - diagnostic médical de SCM } \\
\text { - race/ethnie : ni blanc, ni noir, ni } \\
\text { hispanique } \\
\text { - faibles revenus } \\
\text { - incapacité de travail } \\
\text { - perception du risque et } \\
\text { sèche-cheveux ou lignes HT }\end{array}$ & $\begin{array}{l}\text { Pas de relation avec le sexe } \\
\text { - pas de relation avec le statut } \\
\text { allergique } \\
\text { - différence avec SCM : } \\
\text { HE = moins de femmes, } \\
\text { moins d'asthme, } \\
\text { moins de cas }\end{array}$ \\
\hline $\begin{array}{l}\text { Hillert et al. } \\
2002 \mathrm{a}\end{array}$ & $\begin{array}{l}\text { - Question } \\
\text { - } 1997\end{array}$ & $\begin{array}{l}\text { - Stockholm county } \\
\text { - } n=15000 \\
\text { - } \mathrm{TR}=73 \%\end{array}$ & $\begin{array}{l}\cdot 1,5 \% \mathrm{HE} \\
(n=167 / 10670) \\
\cdot 23,2 \% \text { asthme et } \\
\text { rhume des foins } \\
(n=2520 / 10670)\end{array}$ & $\begin{array}{l}\text { - sexe (1,8\% femmes vs } 1,1 \% \\
\text { d'hommes) } \\
\text { - âge : entre } 60 \text { et } 80 \text { ans (sauf si } \\
\text { suppression des gens avec une } \\
\text { intolérance des amalgames), } \\
\text { - allergies (asthme, rhume des foins, } \\
\text { conjonctivite allergique) et autres } \\
\text { hypersensibilités (amalgames, } \\
\text { nickel...), } \\
\text { - rhumes et hypertension plus } \\
\text { fréquents } \\
\text { - naissance en-dehors des pays } \\
\text { nordiques } \\
\text { - faibles revenus } \\
\text { - non employés (pension ou maladie) }\end{array}$ & $\begin{array}{l}\text { Pas de profil symptomatique } \\
\text { spécifique } \\
\text { - symptômes = } \\
\text { 1) neurovégétatifs (fatigue, céphalées, } \\
\text { difficultés de concentration...) } \\
\text { 2) dermatologiques, oculaires et } \\
\text { respiratoires (irritation oculaire, nez } \\
\text { bouché, odorat altéré, toux...) }\end{array}$ \\
\hline
\end{tabular}

Dans leur étude de suivi de 200 patients présentant des symptômes dermatologiques en relation avec l'utilisation d'écran d'ordinateur et 50 patients hypersensibles à l'électricité décrits comme présentant des symptômes multiples évoqués par l'exposition à divers environnements électriques, Stenberg et al., (2002) décrivent un nombre de symptômes plus important, une incapacité à travailler beaucoup plus fréquente, et donc un moins bon pronostic, particulièrement chez les femmes hypersensibles à l'électricité. Ces deux groupes de patients présentent également une fréquence symptomatique supérieure à celle de la population générale. Les patients hypersensibles à l'électricité présentent plus souvent un échauffement et une sensation de picotement de la face, une sensation de fatigue et des céphalées ainsi que des palpitations que les patients qui se plaignent de dermatite de l'écran qui eux présentent davantage un érythème. Si le facteur évoqué par les patients dans la genèse de leur symptomatologie est dans deux tiers des cas l'usage d'ordinateur et de lampes fluorescentes pour les patients hypersensibles à l'électricité, il est évoqué chez $96 \%$ des patients présentant une dermatite de l'écran. Les pathologies respiratoires atopiques de type asthme ou rhinite allergique ne semblent pas être un facteur de risque de l'HE ou de la dermatite de l'écran alors que l'eczéma constitutionnel semble être un facteur de risque pour les réactions dermatologiques faciales aux expositions environnementales étant donné une plus forte prévalence de der- matite atopique observée dans le groupe des patients se plaignant de dermatite de l'écran.

\section{4. Étiologies de l'hypersensibilité à l'électricité}

\subsection{Quelle relation entre l'hypersensibilité et l'exposition aux champs électromagnétiques?}

\subsubsection{Les études épidémiologiques}

Peu d'études portent sur la relation entre les symptômes et l'exposition aux champs électromagnétiques. Aucune association significative entre les plaintes dermatologiques liées au travail sur écran et l'exposition aux champs magnétiques n'a été observée (Bergqvist et Wahlberg, 1994, Sandström et al., 1995). Seule une étude a pu établir des corrélations entre le champ électrique ambiant (et non celui qui est émis par l'ordinateur) et les symptômes évoqués (Stenberg et al., 1995). La plupart des études ne permettent pas d'associer les symptômes à l'intensité des émissions électromagnétiques des écrans mais simplement à l'usage de ces écrans, notamment à leur durée d'utilisation, le faible taux d'humidité ambiant et la perception de l'activité de l'écran en terme d'émission électromagnétique.

\subsubsection{Les études expérimentales}

Étant donné que les personnes présumées hypersensibles à l'électricité attribuent leurs symptômes à des équipements 
électriques en fonctionnement, des recherches ont été entreprises afin de déterminer si les champs électromagnétiques pouvaient induire ces symptômes et si les personnes pouvaient percevoir ces champs. En effet, la perception d'une relation temporelle entre l'utilisation des appareils électriques incriminés et le développement de symptômes n'implique pas nécessairement que les champs électriques, magnétiques ou électromagnétiques soient responsables des effets observés. Ces études, appelées études de provocation, consistent à exposer les personnes, dans des conditions de double insu, à des situations d'exposition où les champs sont tantôt réellement produits, tantôt de manière simulée et d'analyser leurs réactions en termes de détection du champ, de réactions dermatologiques et /ou de symptômes. Un groupe témoin sert également souvent de point de comparaison pour les paramètres observés. Ces études impliquent que les symptômes soient transitoires et disparaissent généralement peu de temps après l'arrêt de l'exposition, ce qui est généralement le cas. Les études de provocation sont résumées dans le Tableau 2.

La plupart des études de «provocation » ont été réalisées en Suède ou en Norvège, essentiellement chez des personnes qui se plaignent de symptômes dermatologiques en relation avec le travail sur écran (Andersson et al., 1996 ; Flodin, et al., 2000 ; Hamnerius et Sjöberg, 1994 ; Hamnerius et al., 1994 ; Lonne-Rahm et al., 2000 ; Oftedal, Wistnes, et Rygge, 1995 ; Oftedal, et al., 1999 ; Skulberg et al., 2001 ; Swanbeck et Bleeker, 1989 ; Wennberg, et al., 1994). Les personnes ne sont pas capables, même lorsque l'étude est réalisée sur le lieu de travail ou directement avec l'appareil incriminé, de dire si l'appareil, en l'occurrence l'ordinateur, est allumé ou non, lorsque tout indice visuel ou auditif est soigneusement contrôlé.

D'autres études plus récentes se rapportent à l'hypersensibilité aux champs électriques et/ou magnétiques à la fréquence industrielle $(50 \mathrm{~Hz})$ (Lyskov, Sandström, et Hansson Mild, 2001a ; Müeller, et al., 2002 ; Toomingas, 1996 ; Rea, 1991 ; Wang, et al., 1994). Dans certaines publications, les études de provocation ont été utilisées dans un cadre thérapeutique (Andersson et al., 1996 ; Toomingas, 1996) études qui soulignent par ailleurs l'efficacité des thérapies cognitivocomportementales. À une exception près (Rea et al., 1991), ces études de provocation ne permettent pas d'établir un lien entre l'exposition aux champs électriques, magnétiques ou électromagnétiques et les symptômes présentés par les personnes présumées hypersensibles à l'électricité. Dans son étude préliminaire, Rea et al. ont proposé, en simple insu, à 100 personnes traitées pour des problèmes de sensibilité environnementale (SCM et HE) des expositions à des champs électromagnétiques de 2,9 $\mu \mathrm{T}$ (au niveau du sol) à $0,35 \mu \mathrm{T}$ (au niveau de la taille) à des fréquences allant de 0,1 à $5 \mathrm{MHz}$. Sur 100 personnes, 25 réagissent à l'exposition dans une deuxième série d'essais, 16 d'entre elles réagissent à nouveau à l'exposition par des symptômes neurologiques, cardiologiques et respiratoires dans un essai où elles sont comparées à un groupe témoin, en double insu. L'imprécision des données et l'incapacité à reproduire ces résultats (Wang, et al., 1994) ne permettent pas de tirer des conclusions à partir de cette étude réalisée avec une forme d'onde particulière (ondes carrées).

Trois études ont été réalisées pour tester la sensibilité des personnes dites hypersensibles aux radiofréquences générées par les téléphones cellulaires. Une première étude a été réalisée par Johansson (1995) mais n'existe que sous la forme d'un rapport rédigé en suédois. Dans cette étude relatée dans le rapport de Bergqvist et Vogel 1997, seulement une des sept personnes a pu identifier si le téléphone mobile était en marche ou à l'arrêt. L'étude de Hietanen, et al., 2002, n'a pas pu mettre en évidence d'association entre les symptômes exprimés par les 20 personnes hypersensibles testées et l'exposition à deux types de téléphones mobiles (Global System for Mobile Communication (GSM) $902 \mathrm{MHz}$ et $1800 \mathrm{MHz}$ et NMT $900 \mathrm{MHz}$ ). Le nombre de symptômes rapportés était plus élevé pendant l'exposition simulée que pendant les conditions d'exposition réelle et aucune personne n'a pu distinguer l'exposition réelle de l'exposition simulée. Ces résultats mènent à conclure que les symptômes ou sensations ressenties ne sont pas produites par les téléphones cellulaires. Cependant, une étude récente (Swanborn et al., 2003) indique une relation entre le bien-être et les résultats à des tests cognitifs et l'exposition à des champs électromagnétiques tels qu'ils sont produits par les système de mobilophonie $(900 \mathrm{MHz}$, $1800 \mathrm{MHz}$ et $2100 \mathrm{MHz}$ ou «UMTS-like »). Les résultats de cette étude ne permettent pas de savoir si les effets sont indépendants de la perception des champs électromagnétiques émis et les comparaisons du groupe de personnes hypersensibles avec le groupe témoin sont rendues caduques par des problèmes d'appariement pour l'âge et le sexe des deux groupes. Cette étude, subsidiée par le gouvernement hollandais, mérite cependant d'être reproduite.

Globalement, les études de provocation indiquent que les personnes « hypersensibles à l'électricité »n'ont pas été capables de détecter des champs électriques ou magnétiques à des niveaux correspondants à des situations où elles réagissent. $\mathrm{Si}$ ces personnes réagissent dans ces études de provocation, leurs réactions ne sont pas liées aux champs mais à la croyance que le champ est généré ( « $\mathrm{ON}$ ») en référence potentielle à un effet d'attente. Les symptômes se présentent en effet lorsque les personnes qui se plaignent d'HE disent percevoir l'exposition aux champs, indépendamment de la situation réelle d'exposition (réelle ou simulée). En conclusion, la relation entre les champs électriques et magnétiques et les symptômes ressentis n'a pu être démontrée jusqu'à présent.

Ces résultats nourrissent des discussions quant à plusieurs facteurs : le recrutement des personnes testées, les situations de test et l'éventualité de perturbations par d'autres facteurs. Il est possible que les champs ne soient pas adéquatement choisis en termes de durée, d'intensité, de fréquence et que les intervalles entre les sessions d'exposition réelle et simulée soient trop courts. Les symptômes n'apparaissent pas nécessairement immédiatement (bien que ce critère soit souvent pris en compte dans la sélection des sujets) ou systématiquement. Une étude de provocation est une situation artifi- 
Tableau 2

Études de provocation chez des personnes hypersensibles à l'électricité

\begin{tabular}{|c|c|c|c|c|}
\hline Auteurs & Recrutement & Exposition & Mesures & Réponse \\
\hline \multicolumn{5}{|c|}{ Écrans de visualisation } \\
\hline $\begin{array}{l}\text { Swanbeck et Bleeker, } \\
1989 \\
\text { Double insu }\end{array}$ & $\begin{array}{l}n=30 \text { patients avec dermatite } \\
\text { de l'écran }\end{array}$ & $\begin{array}{l}\text { Ordinateur avec CE statique } \\
\text { très important et } \\
\text { ordinateur avec CE statique et } \\
\mathrm{CM} \text { pratiquement nuls } \\
\text { • variation humidité ( } 25 \% \text { et } \\
60 \% \text { ) } \\
3 \text { heures }\end{array}$ & - réactions dermatologiques & $\begin{array}{l}\text { Picotements et démangeaisons } \\
\text { indépendamment du CEM, } \\
\text { symptômes plus marqués si } \\
\text { humidité faible }\end{array}$ \\
\hline $\begin{array}{l}\text { Hamnerius et Sjöberg, } \\
1994\end{array}$ & $n=7$ & $\begin{array}{l}\text { Générateur, Exposition réelle } \\
\text { et simulée, } 3 \text { séries de tests } \\
\text { avec } \mathrm{CM} \text { croissant } \\
8 \times 1 \text { heure/phase } \\
1 \times / \text { jour }\end{array}$ & $\begin{array}{l}\text { - détection des champs } \\
\text { - symptômes } \\
\text { - température de la peau }\end{array}$ & Pas de relation avec CEM \\
\hline $\begin{array}{l}\text { Hamnerius et al., } 1994 \\
\text { Double insu }\end{array}$ & $\begin{array}{l}n=30 \text { patients avec dermatite } \\
\text { de l'écran }\end{array}$ & $\begin{array}{l}\text { Générateur CE et CM, } 3 \text { séries } \\
\text { de fréquences, expo réelle et } \\
\text { simulée } \\
1 \text { heure }\end{array}$ & $\begin{array}{l}\text { - détection des champs } \\
\text { - symptômes } \\
\text { - température de la peau } \\
\text { - rythme cardiaque } \\
\text { - pression artérielle } \\
\text { - réponse électrodermale }\end{array}$ & $\begin{array}{l}\text { - pas de détection des champs, } \\
\text { symptômes non reliés aux } \\
\text { CEM }\end{array}$ \\
\hline $\begin{array}{l}\text { Wennberg, Franzen, et } \\
\text { Paulsson, } 1994 \\
\text { Groupe témoin }(n=13)\end{array}$ & $\begin{array}{l}n=25(15 \mathrm{~F}, 10 \mathrm{H}) \\
\text { Âge moyen }=43 \text { ans }\end{array}$ & $\begin{array}{l}\text { Générateur de CEM : } 50 \mathrm{~Hz} \text {, } \\
20 \mathrm{kHz} \text { et CEM OFF } \\
40 \text { minutes }\end{array}$ & $\begin{array}{l}\text { - détection } \\
\text { - symptômes } \\
\text { - température de la peau du } \\
\text { visage }\end{array}$ & $\begin{array}{l}\text { - pas de détection } \\
\text { - différences de température } \\
\text { plus importantes chez sujets } \\
\text { HE mais indépendante de } \\
\text { l'exposition }\end{array}$ \\
\hline $\begin{array}{l}\text { Andersson et al., } 1996 \\
\text { Double insu } \\
\text { Groupe témoin }\end{array}$ & $\begin{array}{l}n=17(12 \mathrm{~F}, 5 \mathrm{H}) \\
\text { Âge moyen }=42 \text { ans } \\
\text { Tests de provocation avant et } \\
\text { après traitement } \\
\text { psychologique ( } 20 \text { semaines) } \\
+ \text { test ouvert champ ON }\end{array}$ & $\begin{array}{l}2 \text { sessions (1champ } \\
\text { ON/1 champ OFF) } \\
\text { CEM générés par un } \\
\text { ordinateur } \\
30 \text { minutes } \\
1 \text { semaine d'intervalle }\end{array}$ & $\begin{array}{l}\text { - détection des champs } \\
\text { - symptômes } \\
\text { - bilan sanguin (hormones de } \\
\text { stress) }\end{array}$ & $\begin{array}{l}\text { Pas de détection, réaction plus } \\
\text { intense quand jugement que le } \\
\text { champ est ON, indépendante } \\
\text { de la présence réelle du CEM }\end{array}$ \\
\hline $\begin{array}{l}\text { Oftedal, Vistnes, et } \\
\text { Rygge, } 1995 \\
\text { Double insu }\end{array}$ & 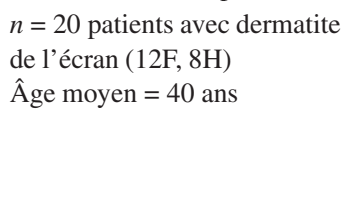 & $\begin{array}{l}\text { Usage de filtres } \\
\text { électromagnétiques, } \\
2 \text { semaines sans filtre, } \\
2 \text { semaines avec filtre inactif, } \\
2 \text { semaines avec filtre actif } \\
\text { Sur lieu de travail }\end{array}$ & - symptômes & $\begin{array}{l}\text { Réduction des symptômes } \\
\text { avec filtres, plus prononcée } \\
\text { avec filtres actifs }\end{array}$ \\
\hline $\begin{array}{l}\text { Oftedal, Nyvang, et } \\
\text { Moen, } 1999 \\
\text { Double insu }\end{array}$ & $\begin{array}{l}n=42(33 \mathrm{~F}, 9 \mathrm{H}) \\
\hat{\text { Âge moyen }}=41 \text { ans }\end{array}$ & $\begin{array}{l}\text { Usage de filtres } \\
\text { électromagnétiques, } 1 \text { semaine } \\
\text { sans filtre, } \\
3 \text { mois avec filtre inactif, } \\
3 \text { mois avec filtre actif } \\
\text { Sur lieu de travail }\end{array}$ & • symptômes & $\begin{array}{l}\text { Réduction significative des } \\
\text { symptômes pendant périodes } \\
\text { de filtre (effet visuel ou } \\
\text { psychologique), pas de } \\
\text { différence si filtre actif ou } \\
\text { inactif }\end{array}$ \\
\hline $\begin{array}{l}\text { Flodin, Seneby, et } \\
\text { Tegenfeldt, } 2000 \\
\text { Double insu } \\
\text { Groupe témoin }\end{array}$ & $\begin{array}{l}n=15(11 \mathrm{~F}, 4 \mathrm{H}) \\
\text { Âge moyen }=48 \text { ans }\end{array}$ & $\begin{array}{l}4 \text { sessions ; } 2 \text { réelles, } \\
2 \text { simulées } \\
\text { Ordinateur } \\
\text { Intervalles de plusieurs jours } \\
\text { Jusqu'à } 1 \text { heure } \\
\text { À la maison ou sur le lieu de } \\
\text { travail }\end{array}$ & $\begin{array}{l}\text { - détection des champs } \\
\text { - symptômes }\end{array}$ & $\begin{array}{l}\text { Pas de différence de détection } \\
\text { avec groupe témoin, } \\
\text { Pas de différence } \\
\text { symptômatique }\end{array}$ \\
\hline $\begin{array}{l}\text { Lonne-Rahm et al., } 2000 \\
\text { Double insu } \\
\text { Groupe témoin toujours } \\
\text { exposé }\end{array}$ & $n=24$ & $\begin{array}{l}\text { Stress et CEM } \\
\text { Stress sans CEM } \\
\text { CEM sans stress } \\
\text { Ordinateur } \\
30 \text { minutes } \\
\text { Stress = tâche cognitive }\end{array}$ & $\begin{array}{l}\text { - détection des CEM } \\
\text { - bilan sanguin (facteurs de } \\
\text { stress) } \\
\text { - biopsies de la peau }\end{array}$ & $\begin{array}{l}\text { - augmentation des } \\
\text { symptômes dermatologiques } \\
\text { quand les personnes se savent } \\
\text { exposées ou croient être } \\
\text { exposées } \\
\text { - pas de différence entre CEM } \\
\text { ON ou OFF, } \\
\text { d'où pas de réaction aux CEM }\end{array}$ \\
\hline $\begin{array}{l}\text { Skulberg et al., } 2001 \\
\text { Double insu } \\
\text { Groupe témoin }(n=58)\end{array}$ & $n=59$ & $\begin{array}{l}\text { Réduction champ électrique } \\
\text { statique de l'ordinateur } \\
\text { Sur lieu de travail }\end{array}$ & $\begin{array}{l}\text { - symptômes après } 2 \text { semaines } \\
\text { et } 4 \text { semaines après } \\
\text { l'intervention }\end{array}$ & Réduction des plaintes faciales \\
\hline
\end{tabular}


Tableau 2

(suite)

\begin{tabular}{|c|c|c|c|c|}
\hline Auteurs & Recrutement & Exposition & Mesures & Réponse \\
\hline \multicolumn{5}{|c|}{ Radiofréquences (GSM) } \\
\hline $\begin{array}{l}\text { Hietanen, Hämäläinen, } \\
\text { et Husman, } 2002 \\
\text { Double insu }\end{array}$ & $n=20(17 \mathrm{~F}, 7 \mathrm{H})$ & $\begin{array}{l}\text { - téléphone cellulaire } \\
\text { NMT ( } 900 \mathrm{MHz}) \\
\text { GSM }(900 \mathrm{MHz}) \\
\text { GSM }(1800 \mathrm{MHz}) \\
30 \text { minutes } \\
3 \text { à } 4 \text { sessions par jour }\end{array}$ & $\begin{array}{l}\text { - détection des champs } \\
\text { - symptômes } \\
\text { - pression sanguine } \\
\text { - rythme cardiaque } \\
\text { - fréquence respiratoire }\end{array}$ & $\begin{array}{l}\text { - pas de détection des champs, } \\
\text { - symptômes divers dans } \\
\text { région céphalique plus } \\
\text { élevés pendant exposition } \\
\text { simulée }\end{array}$ \\
\hline \multicolumn{5}{|c|}{ Fréquence du réseau $(50-60 \mathrm{~Hz})$} \\
\hline $\begin{array}{l}\text { Rea, } 1991 \\
\text { Simple insu pour phase } \\
1 \text { et } 2 \text {, double insu pour } \\
\text { phase } 3 \\
\text { Groupe témoin }\end{array}$ & $\begin{array}{l}\text { Phase } 1: n=100 \\
\text { Phase } 2: n=25 \\
\text { Phase } 3: \mathrm{n}=16\end{array}$ & $\begin{array}{l}\text { Générateur ondes carrées de } \\
0,1 \text { à } 5 \mathrm{MHz}(70 \mathrm{nT} \text { à } \\
2900 \mathrm{nT}) \text {, expositions } \\
\text { simulées et réelles, } \\
\text { Tests de } 3 \text { minutes }\end{array}$ & $\begin{array}{l}\text { - symptômes } \\
\text { • paramètres physiologiques }\end{array}$ & $\begin{array}{l}16 \text { personnes sur } \\
100 \text { répondent de manière } \\
\text { répétée à certaines fréquences } \\
\text { par des symptômes } \\
\text { essentiellement neurologiques }\end{array}$ \\
\hline $\begin{array}{l}\text { Wang, Hawkins, et Rea, } \\
1994 \\
\text { Groupe témoin }(n=34)\end{array}$ & $n=47$ patients $\mathrm{SCM}^{\mathrm{a}}$ et $\mathrm{HE}$ & $\begin{array}{l}3 \text { expo simulées, } 6 \text { expo } \\
\text { réelles }(10 \mathrm{~Hz}, 50 \mathrm{~Hz}, 60 \mathrm{~Hz} \text {, } \\
100 \mathrm{~Hz}, 200 \mathrm{~Hz}, 300 \mathrm{~Hz}) \\
3 \text { minutes par test }\end{array}$ & $\begin{array}{l}\text { • symptômes } \\
\text { • paramètres physiologiques }\end{array}$ & $\begin{array}{l}\text { Pas de différence avec expo } \\
\text { simulée }\end{array}$ \\
\hline Toomingas, 1996 & 1 patient de 37 ans & $\begin{array}{l}\text { Générateur de CM } 50 \mathrm{~Hz} \\
(34 \mu \mathrm{T} \text { ou } 100 \mu \mathrm{T}), 24 \text { séances } \\
\text { de } 1 \text { ou } 10 \text { secondes. }\end{array}$ & $\begin{array}{l}\text { - détection } \\
\text { - symptômes }\end{array}$ & Pas de relation avec $\mathrm{CM}$ \\
\hline $\begin{array}{l}\text { Lyskov, Sandström, et } \\
\text { Hansson Mild, } 2001 \\
\text { Groupe témoin }(n=20)\end{array}$ & $\begin{array}{l}n=20(15 \mathrm{~F}, 5 \mathrm{H}) \\
\text { Âge moyen }=46 \text { ans }\end{array}$ & $\begin{array}{l}\text { Générateur de CM } 60 \mathrm{~Hz} \\
(10 \mu \mathrm{T}), 1 \text { expo réelle, } 1 \text { expo } \\
\text { simulée } \\
10 \text { minutes }\end{array}$ & $\begin{array}{l}\text { Détection, EEG, PEV, activité } \\
\text { électrodermale, } \\
\text { ECG, pression sanguine, test } \\
\text { d'arithmétique }\end{array}$ & $\begin{array}{l}\text { Pas de détection des champs, } \\
\text { différences dans la ligne de } \\
\text { base pour le rythme cardiaque } \\
\text { et l'activité électrodermale }\end{array}$ \\
\hline $\begin{array}{l}\text { Mueller, Krueger, et } \\
\text { Schierz, } 2002 \\
\text { Double insu } \\
\text { Groupe témoin }(n=14)\end{array}$ & $n=49(30 \mathrm{~F}, 19 \mathrm{H})$ & $\begin{array}{l}\text { Générateur de CM et CE } \\
50 \mathrm{~Hz}(6 \mu \mathrm{T}, 100 \mathrm{~V} / \mathrm{m})\end{array}$ & $\begin{array}{l}\text { - détection } \\
\text { - symptômes }\end{array}$ & $\begin{array}{l}\text { Résultats significatifs pour } \\
\text { détection pour } 7 \text { personnes, } \\
\text { pas de différence entre HE et } \\
\text { contrôles pour détection ou } \\
\text { symptômes }\end{array}$ \\
\hline
\end{tabular}

${ }^{\text {a }} \mathrm{SCM}=$ syndrome chimique multiple.

cielle, souvent moins complexe que la situation réelle, sans interaction avec d'autres facteurs précipitants potentiels (physiques, chimiques, psychosociaux, etc.) et avec seulement une source d'exposition. En revanche, elle permet d'isoler la composante champ des autres facteurs et de la tester spécifiquement, dans des conditions contrôlées. La vie réelle implique des expositions à de multiples sources de champs électromagnétiques, à différentes fréquences, formes d'onde, intensités et variations temporelles. De nombreuses combinaisons sont possibles sans que l'on sache laquelle pourrait être la plus susceptible d'induire des symptômes d'hypersensibilité chez une personne particulière. Il est important d'établir correctement les critères d'inclusion et de bien caractériser les personnes hypersensibles qui entrent dans l'étude, de tester la capacité des personnes à réagir au système mis en place dans des conditions tests de champ ON et de réfléchir soigneusement aux caractéristiques de l'exposition choisie pour l'étude. La complexité de ces choix croît si l'on considère la forte hétérogénéité reconnue au niveau de l'HE.

Certains chercheurs ont également proposé des tâches cognitives aux sujets hypersensibles pendant les tests de provocation dans le but de proposer des situations de stress ou de tester si les effets des champs électromagnétiques peuvent apparaître ou être plus prononcés chez les personnes hypersensibles par rapport au groupe témoin (Lonne-Rahm et al., 2000 ; Trimmel et Schweiger, 1998 ; Lyskov et al., 2001). Lyskov et al., (2001) n'observent aucun effet de l'exposition intermittente à un champ magnétique de $10 \mu \mathrm{T}, 60 \mathrm{~Hz}$, sur la performance à un test d'arithmétique. Hypersensibles et sujets contrôles se distinguent en revanche quant à leurs caractéristiques physiologiques de base (activité électrodermale, rythme cardiaque) suggérant pour les premiers une prédisposition physiologique à la sensibilité aux stresseurs environnementaux physiques et psychosociaux. Dans l'étude de LonneRahm et al., (2000), un test visuel est combiné à un test arithmétique afin d'induire une situation de stress. Un accroissement de symptômes est observé lorsque les personnes savent ou croient être exposées aux champs électromagnétiques (écran d'ordinateur). En revanche, dans des conditions de double insu, aucune différence n'est observée entre les situations de champ réelles et simulées. Trimmel et Schweiger (1998) observent quant à eux une détérioration de l'attention, de la capacité perceptive et des performances mnésiques à la suite d'une exposition de volontaires à des champs magnétiques de $1000 \mu \mathrm{T}$ associés à un bruit de $45 \mathrm{~dB}$. Parmi les sujets, ceux dont la sensibilité subjective (réponse oui à la question : 
«Pensez-vous que vous appartenez à un groupe de personnes qui réagissent de manière exceptionnelle aux champs électromagnétiques ») est plus prononcée ont des résultats plus détériorés. Selon Hillert (2001), on peut faire l'hypothèse que les champs électriques ou les champs magnétiques ne sont ni suffisants ni nécessaires pour entraîner les symptômes rapportés. En effet, ces symptômes peuvent apparaître en l'absence de champ électromagnétique (par exemple pendant les expositions simulées) ou lorsque l'étude n'est pas réalisée en double insu, comme une réponse à un stimulus conditionné, ou encore être la manifestation d'une pathologie physique. Les champs électromagnétiques ne sont dans ce cas pas la cause nécessaire des symptômes. D'un autre côté, l'exposition aux champs électromagnétiques ne provoque pas toujours des symptômes pendant les études de provocation, des améliorations de la symptomatologie sont parfois observées sans évitement de la source de champs électromagnétiques et dans ce sens, les champs électromagnétiques ne sont pas une cause suffisante à l'apparition des symptômes (Hillert, 2001 ; Flodin, et al., 2000). Quoi qu'il en soit, dans la plupart des études réalisées, les résultats indiquent l'incapacité des personnes « hypersensibles à l'électricité » à détecter les champs et/ou à réagir spécifiquement à ceux-ci en termes de symptômes. Les personnes présentent souvent des symptômes lors de ces tests de provocation mais ils apparaissent indépendamment des conditions d'exposition (réelle ou simulée). Un lien entre l'exposition et l'HE n'a donc pu être démontré indépendamment de la conscience de la présence du champ. Cette constatation conduit Leitgeb et Schröttner (2003) à distinguer la sensibilité électromagnétique et l'HE. La première consisterait en la capacité de percevoir des expositions à des champs électromagnétiques sans nécessairement développer des symptômes. L'HE se rapporterait au développement de symptômes causés par les expositions aux champs électromagnétiques de notre environnement, sans que cela implique une relation causale démontrée. Ce terme serait utilisé par les patients qui associent leurs symptômes aux sources de champs électromagnétiques et par les médecins qui nomment ainsi un syndrome comprenant des symptômes non spécifiques dont l'origine est encore inconnue. De là, on pourrait s'attendre à ce que les patients hypersensibles à l'électricité soient également plus sensibles aux champs électromagnétiques. Cela reste à démontrer.

\subsection{Une origine multifactorielle}

S'il n'est pas possible d'exclure le rôle contributif des champs électromagnétiques dans l'HE, il n'existe actuellement que trop peu d'indications d'un tel rôle. À côté des phénomènes électriques, d'autres facteurs ont été évoqués dans l'HE, comme la charge de travail élevée, une situation psychosociale défavorable mais aussi des réactions psychophysiologiques individuelles au stress qui indiqueraient une réaction exagérée du système nerveux central et autonome, sans conclusion définitive à ce sujet. Ces facteurs ont été plus particulièrement appréhendés dans le cas des symptômes liés aux écrans. Il s'agit de facteurs physiques, chimiques, organisationnels, psychosociaux et individuels.

\subsubsection{Hypersensibilité et autres facteurs physiques}

Les facteurs physiques autres que les champs électromagnétiques et notamment les champs électriques ambiants sont souvent cités comme facteurs associés aux plaintes dont le faible niveau d'humidité, inférieur à $30 \%$ (Bergqvist et Wahlberg., 1994 ; Liden et Wahlberg, 1985) et la température ambiante élevée, supérieure à $23^{\circ}$ (Jorulf et al., 1993), particulièrement pour les réactions dermatologiques légères en relation avec les écrans. Les lumières clignotantes (flickering light) et les tubes fluorescents (Bergqvist, 1995) semblent également jouer un rôle. En effet, les modulations lumineuses sont considérées comme un facteur susceptible d'affecter le système nerveux central et/ou autonome des personnes dites hypersensibles (Sandström et al., 1997). Citons également les relations entre la poussière et les champs électriques statiques, susceptibles d'expliquer les symptômes oculaires et dermatologiques, même si aucune preuve scientifique n'a pu être apportée (Stenberg et al., 1995).

\subsubsection{Hypersensibilité et facteurs chimiques}

L'association, in vitro, entre l'exposition aux champs magnétiques émis par les ordinateurs et la libération de vapeur de mercure à partir d'amalgames dentaires (Örtendahl, et al., 1991) a renforcé l'hypothèse d'un lien entre les amalgames dentaires et les problèmes d'HE. Cependant, aucune augmentation de libération de vapeur de mercure n'a pu être démontrée chez ces personnes (Bergdahl, et al., 1998). L'intolérance aux amalgames dentaires associée à l'HE est fréquemment rapportée (56\% des personnes HE interrogées, $n=103$ ) par les personnes HE, avec une amélioration de la symptomatologie chez $29 \%$ des personnes après retrait de ces amalgames (Bergdahl, et al., 1994). S'il existe un chevauchement au niveau de la prévalence de personnes qui se décrivent comme hypersensibles aux champs électriques et magnétiques et intolérantes aux amalgames (Hillert, 2002a), aucun effet combiné de ces deux facteurs n'a pu être démontré jusqu'à présent. Par ailleurs, l'intervention de facteurs explicatifs d'ordre psychologique est également soulignée dans les symptômes rapportés par les personnes qui se plaignent d'effets sur la santé liés aux amalgames dentaires (Bailer et al., 2001). D'autres agents chimiques tels que les retardateurs de flamme présents dans les ordinateurs et autres appareils électroniques font également partie des facteurs cités dans l'origine de l'HE, sans qu'aucune relation causale n'ait été démontrée actuellement. Le diagnostic de sensibilité chimique multiple apparaît comme un prédicteur de l'HE dans certaines études (Levallois, 2002). L'hypothèse de réactions causées par des mycotoxines comme explication de l'HE a également été posée (Antilla, 2000).

\subsubsection{Hypersensibilité et facteurs organisationnels et psychosociaux}

Les études réalisées sur les symptômes dermatologiques chez les travailleurs sur écran indiquent quelques facteurs 
associés à l'apparition et/ou le maintien de symptômes tels que la charge de travail perçue, le faible contrôle perçu sur le travail, le manque de support social de la part des collègues ou des supérieurs, l'insatisfaction au travail, une réorganisation du travail, l'introduction rapide de nouvelles routines ou de nouveaux équipements mais également des problèmes familiaux (Eriksson et al., 1997b ; revue in Hillert et Kolmodin-Hedman, 1997) et de manière générale, les conditions de travail source de stress (Bergqvist et Wahlberg, 1994 ; Bergqvist, 1995 ; Berg et al., 1992). C'est pourquoi, les facteurs de stress psychosociaux issus de l'environnement professionnel peuvent être considéré comme un indicateur plausible de risque à long terme (Eriksson et al., 1997a, 1997b).

Parmi les facteurs psychosociaux, relevons également l'importance de la perception des risques et la peur qu'engendrent les nouvelles technologies ainsi que le rôle joué par les médias et les groupes de pression dans la communication et la perception des risques liés à l'électricité. On constate en effet une prévalence accrue de plaintes subjectives liées à la santé chez les personnes qui ont davantage de craintes par rapport aux champs électromagnétiques (McMahan et Meyer, 1995). La crainte du public envers les tumeurs cérébrales en relation avec l'utilisation des GSM illustre de quelle manière des facteurs tels qu'une technologie nouvelle (appareils émettant des ondes peu familières ; les radiofréquences), qui peut atteindre les enfants (question des leucémies infantiles en relation avec l'exposition résidentielle aux champs magnétiques à la fréquence industrielle), qui pourrait provoquer de graves pathologies irréversibles (cancer), dans un climat d'incertitude scientifique, peuvent influencer la perception du risque par le public (revue in Kunsh et al., 1994 ; OMS, 1998 ; Frick, et al., 2002). Lorsque l'exposition est involontaire et/ou qu'elle est régulièrement mise à la une dans ses dangers potentiels sur la santé par les médias, elle est d'autant plus perçue comme un risque pour la santé (Wartenberg et Greenberg, 1992). Il est important de souligner que la perception du risque, en plus du risque lui-même, détermine des changements somatiques et le relevé des symptômes (MacGregor et Fleming, 1996) et peut entraîner une détresse subjective importante (Frick, et al., 2002).

L'HE est-elle une pathologie des croyances ? Les résultats des études de provocation semblent indiquer que les patients hypersensibles à l'électricité répondent à partir de leur croyance au fait d'être exposé plutôt qu'en fonction de leur exposition réelle (Andersson et al., 1996 ; Lonne-Rahm et al., 2000). Il semble important de comprendre les croyances individuelles (pourquoi se considère-t-on comme « inhabituellement sensible »?), le style cognitif (défini comme la manière dont l'individu perçoit et traite l'information) et le style d'attribution des patients ainsi que les croyances de leur groupe de référence en matière de santé et d'environnement (Staudenmayer, 2000). Cela s'applique également au niveau des intervenants, des groupes d'entraide, des cliniques environnementales et des médias en général, qui incitent souvent le patient à se considérer comme victime incomprise des soins de santé traditionnels (Sparks, 2000a).

\subsubsection{Hypersensibilité et facteurs individuels : physiologiques et psychophysiologiques}

Le fait que, à exposition égale, seules certaines personnes développent l'HE implique l'intervention de facteurs internes relatifs à l'individu. Le rôle joué par ces facteurs d'ordre individuel comme facteur de prédisposition à l'hypersensibilité nécessite cependant davantage de recherches. Différents facteurs ont été identifiés parmi lesquels l'âge et le sexe. Les symptômes neurovégétatifs sont plus fréquents chez les personnes hypersensibles plus âgées mais la plupart des personnes hypersensibles sont âgées entre 40 et 50 ans. Les études scandinaves relèvent un nombre de femmes HE plus élevé par rapport à celui des hommes (Hillert et al., 1999 ; Bergdahl, et al., 1994), ce qui contraste avec les données de l'étude californienne de Levallois et al., (2002) réalisée dans la population générale, relatives aux personnes qui se considèrent hypersensibles à l'électricité.

La recherche d'un éventuel marqueur pathophysiologique de cette HE n'a pas encore donné de résultats probants. L'activité des cholinestérases n'est pas réduite dans une étude sur la relation avec la fatigue exprimée par les personnes hypersensibles à l'électricité et l'exposition aux champs électromagnétiques (Hillert et al., 2001a). Les traitements par antioxydants (vitamine $\mathrm{C}$, E et Selenium), destinés à tester l'hypothèse d'un stress oxydatif par l'action des champs électromagnétiques, n'améliorent pas les symptômes ou le statut sérique oxidatif des personnes présumées hypersensibles (Hillert et al., 2001b). Des différences ont été notées au niveau de certains paramètres dermatologiques et histopathologiques, notamment quant au nombre et à la distribution des mastocytes au niveau du derme (Gangi and Johansson, 1997, 2000 ; Johansson et al., 1994) avec des résultats cependant contradictoires (Berg, et al., 1990a ; Berg, et al., 1990b ; Bergqvist et Vogel, 1997). La mélatonine, l'hormone la plus souvent citée dans la problématique des effets sur la santé des champs électromagnétiques (Stevens et Davis, 1996), a très peu été étudiée en relation avec l'HE. Le rapport européen signale, sur la base de résumés pour des congrès et de compterendus de recherches, tantôt des niveaux accrus de mélatonine dans un sous-groupe de patients hypersensibles présentant à la fois des symptômes dermatologiques et neurasthéniques (Wadman et al., 1996 ; en suédois, cité par Bergqvist et Vogel, 1997), tantôt l' absence de différence entre hypersensibles et sujets en bonne santé (Bergqvist et Vogel, 1997).

L'hypothèse que l'HE soit en partie attribuable à des réactions psychophysiologiques au stress liées aux conditions de travail et/ou familiales a également été soulevée. La plupart des études réalisées portent sur des personnes qui se plaignent de symptômes dermatologiques en relation avec le travail sur écran. Une température de la peau plus élevée au niveau du visage est décrite dans l'étude de Wennberg et al., (1994) chez les hypersensibles par rapport au groupe de référence, indépendamment de l'exposition aux champs électriques. Une réactivité différente aux modulations lumineuses apparaît également chez les patients hypersensibles par rap- 
port aux sujets contrôles, les patients hypersensibles présentant des amplitudes plus importantes de potentiels évoqués visuels à toutes les fréquences de stimulation (de 20 à $75 \mathrm{~Hz}$ ) (Wibom et al., 1995 ; cité par Bergqvist et Vogel, 1997 ; Sandström et al., 1997). Lyskov et al., (2001) décrit également des différences au niveau de la ligne de base entre patients hypersensibles et sujets contrôles au niveau du rythme cardiaque (accéléré chez les hypersensibles) et de la réponse électrodermale (augmentée chez les hypersensibles). Des différences apparaissent notamment au niveau de certains dosages hormonaux (prolactine et thyroxine, ACTH) pendant le travail sur écran par rapport aux périodes de repos, résultats interprétés comme une réaction de stress (Berg et al., 1992 ; Arnetz et Berg, 1996). C'est ainsi que la notion de «technostress » a été suggérée pour signifier un état d'éveil mental et physiologique observé chez des employés dont le travail dépend principalement des ordinateurs (Berg et al., 1992 ; Arnetz et Wiholm, 1997). Ce «technostress » survient généralement lorsque l'employé perçoit son travail comme stimulant mais considère simultanément qu'il ne possède pas les habiletés nécessaires. L'hypothèse qui émane de ces données serait que les personnes HE pourraient présenter une hyper-réactivité du système nerveux central et autonome, probablement attribuable à une prédominance sympathique. Elles se caractériseraient par une prédisposition à la sensibilité à différents stresseurs environnementaux physiques et psychosociaux.

\subsubsection{Hypersensibilité et évaluations psychologiques}

Une des caractéristiques soulignée par Hillert et al., (2002a) est le plus haut degré de fréquence et d'intensité des plaintes liées à la santé chez les patients qui se plaignent d'HE, cette caractéristique avait déjà été soulignée dans d'autres rapports (Leitgeb, 1998). D'après Leitgeb, en dehors de cette caractéristique, ces personnes ne diffèrent pas du reste de la population générale. Elles constituent un groupe très hérérogène en termes de scolarité, statut social et économique mais également en termes de symptomatologie.

En général, il apparaît que les patients qui se plaignent d'HE ne sont pas plus déprimés, plus anxieux et ne présentent pas plus de désordres somatoformes que la population générale (Arnetz, 1998). Les patients qui se plaignent d'HE ne présentent généralement pas de pathologie psychiatrique. Cependant, bien que diversifiées en fonction des critères de sélection utilisés, des différences ont été décrites au niveau des profils psychologiques des patients « hypersensibles à l'électricité » avec les groupes témoins, notamment au niveau des échelles de somatisation (Hillert et Kolmodin-Hedman, 1997 ; Hillert et al., 1999). Il est difficile de déterminer si ces scores plus élevés sont à l'origine ou une conséquence de l'HE.

Dans une étude également suédoise, Bergdahl (1995) note des scores plus élevés dans le groupe des patients HE $(n=20)$ que le groupe témoin $(n=20)$ au niveau de différentes échelles de personnalité, de fonctionnement psychologique et de qualité de vie. De plus, les patients qui présentent des symptômes attribués à l'électricité en général ( $\mathrm{EG}, n=10)$ diffè- rent psychologiquement des patients qui attribuent leurs symptômes aux écrans ( $\mathrm{VG}, n=10$ ). Le groupe EG décrit des symptômes plus variés et plus fréquents et évoque davantage les symptômes suivants : fatigue, difficulté de concentration, difficulté à prendre des initiatives, difficulté dans les relations sociales. Le score supérieur obtenu au niveau des échelles d'anxiété somatique, de tension musculaire et de psychasthénie dans le groupe EG souligne leur propension à l'anxiété que chez les patients VG et la nécessité d'une prise en charge différenciée. Le groupe VG se différenciant essentiellement au niveau des échelles de tension musculaire et d'anxiété somatique indique une propension à somatiser en situation de stress. En revanche, les deux groupes présentent communément des symptômes dermatologiques, de la fatigue, des douleurs, des vertiges, des céphalées et des difficultés de concentration.

Dans une autre étude, les mêmes auteurs constatent que les patients présentant des symptômes attribués aux champs électromagnétiques $(n=44)$ apparaissent plus déprimés, plus anxieux (anxiété-état) et plus stressés sur la base d'échelles standardisées (Bergdahl et Bergdahl, 2001). Les niveaux obtenus sont inférieurs à ceux généralement obtenus chez des patients psychiatriques même si $23 \%(n=10)$ des patients présentent des scores supérieurs au seuil (10) à l'échelle de dépression de Beck (BDI). Ces résultats furent interprétés par les auteurs comme la traduction d'un syndrome de somatisation.

\section{5. Étapes de développement de l'hypersensibilité à l'électricité}

Différentes étapes ont été décrites (Bergqvist et Vogel, 1997) conduisant à l'HE. Elle débute par une apparition temporaire de symptômes. Les personnes ayant entendu parler de l'existence de problèmes d'HE éventualisent une relation possible entre l'occurrence de ces symptômes et l'exposition aux champs électromagnétiques. À ce stade, une infirmation et des explications alternatives permettent d'éviter le développement d'une HE ou sont des éléments qui permettent de prédire un bon pronostic.

Dans un second temps, si les symptômes persistent, augmentent en intensité, durée et/ou en nombre, le patient se met à rechercher la confirmation de son hypothèse. Des conduites d'évitement commencent à apparaître ainsi que des changements dans son style de vie.

Quelques personnes atteignent une troisième phase dans laquelle les symptômes neurovégétatifs sont provoqués par la proximité de presque toutes les sources de champs électromagnétiques, convaincues de la relation causale entre ces symptômes et les sources de champ électromagnétique. À ce stade, le pronostic est plutôt mauvais et souvent, seules certaines améliorations dans la vie quotidienne sont obtenues lors de la prise en charge de ces personnes.

Le succès des différentes interventions et le pronostic dépend donc fortement du moment de l'intervention, comme le soulignent Hillert et Kolmodin-Hedman (1997). 


\section{Vers un modèle explicatif de l'hypersensibilité à l'électricité}

Si les symptômes médicalement inexpliqués attribués à l'environnement sont parfois considérés comme des symptômes de somatisation, il est sans doute important de souligner que le manque d'explication médicale ne doit pas nécessairement faire pencher vers une qualification psychiatrique du syndrome (Escobar, et al., 2002).

L'absence de critères objectifs permettant de diagnostiquer l'HE invite certains à la décrire comme une maladie psychosomatique alors que d'autres prétendent qu'il s'agit là d'une pathologie qui trouve ses origines dans un trouble toxicologique d'origine chimique dont l'identification est actuellement impossible étant donné nos techniques de détection ou qui se présente pour des niveaux d'exposition très faibles auxquels ces personnes ont été sensibilisées. Il apparaît cependant qu'un modèle intégratif, de type multidimensionnel serait utile pour la recherche et une meilleure compréhension de l'HE. Dans cette perspective, la Fig. 1 résume les principales caractéristiques du modèle proposé pour illustrer le développement et le maintien de l'HE.

Plusieurs facteurs interviendraient comme facteurs initiateurs de l'HE, des facteurs externes ; physiques (champs élec-

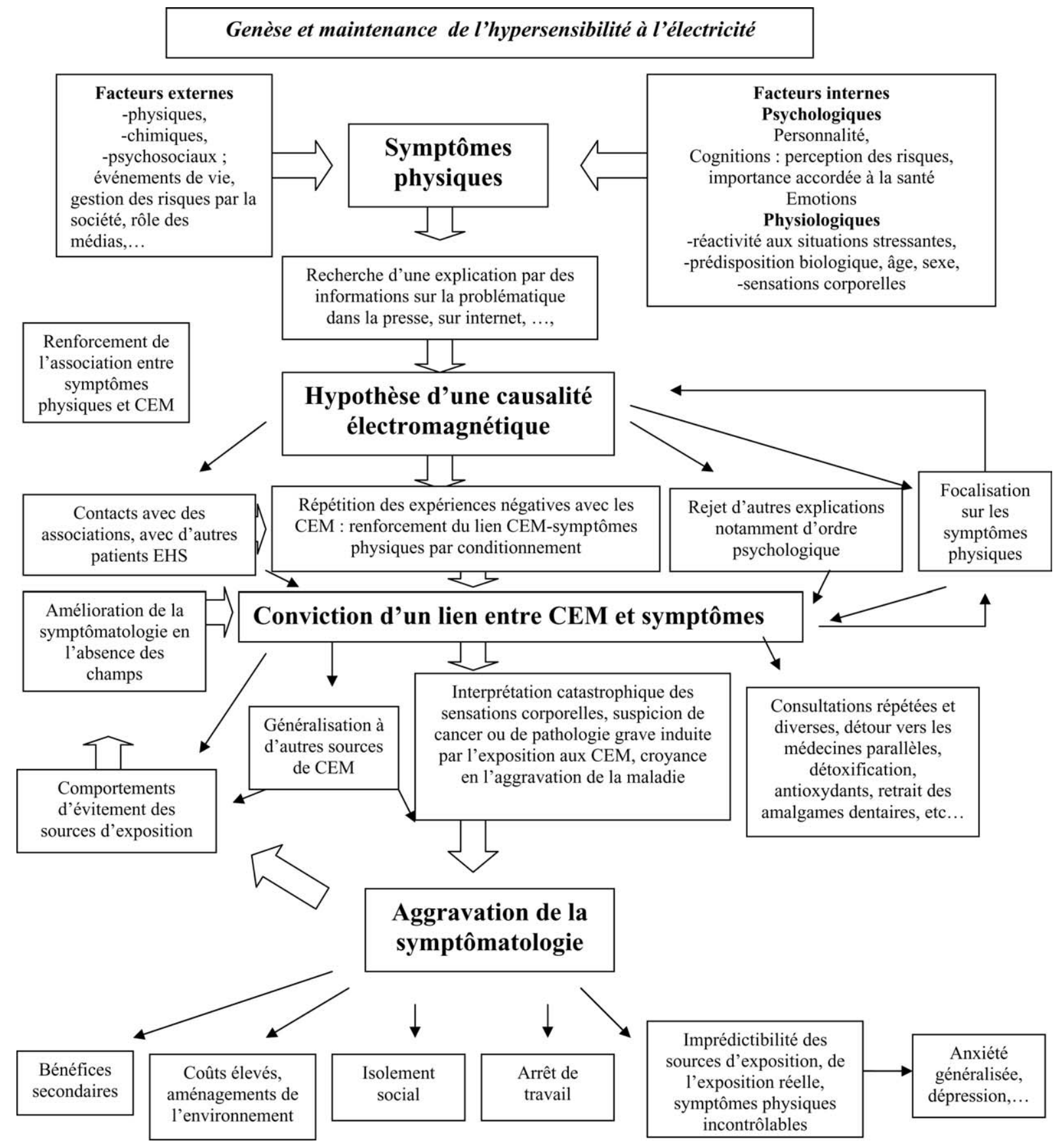

Fig. 1. Proposition d'un modèle explicatif de la genèse et de la maintenance de l'hypersensibilité à l'électricité. 
tromagnétiques, humidité de l'air, modulations lumineuses...), chimiques, psychosociaux (facteurs de stress professionnels, familiaux... événements de vie, manière dont la société et les différentes institutions gèrent les risques, rôle des médias). Des facteurs internes joueraient également, d'ordre psychologique (personnalité, cognitions (style cognitif, croyances en matière de santé, perception des risques, intérêt pour l'environnement, confiance dans les institutions...), émotions et comportements (notamment vis-à-vis de la santé) et d'ordre physiologique (prédisposition ou sensibilité génétique et biologique, réactivité psychophysiologique au stress...). La perception des risques, les informations négatives concernant l'agent environnemental et l'anxiété liée à l'environnement jouent également un rôle important dans l'apparition et l'entretien de ces syndromes, comme l'indiquent certaines études sur le syndrome chimique multiple (Winters et al., 2003). Ajoutons à cela l'attention attribuée par les médias, le manque de confiance dans les institutions, qu'elles soient politiques, scientifiques ou médicales et l'influence des groupes de pression.

Il est en effet possible que des mécanismes autonomes, médiés par le stress ou une sensibilisation comportementale interviennent dans la genèse des symptômes (Kipen et Fiedler, 2002b). Les symptômes ou du moins leur maintien et leur généralisation pourraient ne pas être dus à l'environnement électromagnétique et pourraient n'être que le reflet de l'interprétation individuelle de symptômes qui apparaissent naturellement chez la plupart des personnes et de la manière dont les facteurs de stress environnementaux sont gérés.

Cette reconnaissance d'une multitude de facteurs à l'origine des plaintes exprimées a déjà été soulignée dans d'autres pathologies environnementales similaires dont la sensibilité multiple chimique et notamment par Pennebaker (1994) qui identifie clairement certains facteurs individuels, perceptuels et émotionnels susceptibles d'influencer l'expression de ces plaintes.

Certains facteurs interviendraient dans l'amplification des symptômes, notamment l'hypervigilance, l'attention sélective et la tendance à percevoir les sensations somatiques comme déplaisantes (Barsky et Klerman, 1983). Il s'ensuivrait un cercle vicieux où un événement modeste est attribué à un agent spécifique, en l'occurrence l'électricité. Ces symptômes initiaux se verraient renforcés par une réaction corporelle à l'anxiété et la crainte éprouvée face à la source perçue du problème. Les données concernant les relations entre facteurs de stress et problèmes dermatologiques chez les travailleurs sur écran prônent pour cette hypothèse (Bergqvist, 1995). Le modèle du conditionnement a également été suggéré pour expliquer les intolérances idiopathiques environnementales dont font partie l'HE et le syndrome chimique multiple (Sparks, 2000b).

Face à l'incapacité de la médecine traditionnelle à aider ces patients de par l'absence de critères objectivables ou de traitement éprouvé, les patients hypersensibles multiplient les consultations médicales et se tournent fréquemment vers d'autres pratiques, testent différentes possibilités (détoxifica- tion, retrait des amalgames dentaires) avec plus ou moins de succès au niveau de la symptomatologie exprimée et sans que l'on sache s'il s'agit d'un effet placebo et d'une manière de retrouver une sensation de contrôle pour le patient (Pennebaker, 1994). Par ailleurs, l'entretien et la maintenance des symptômes liés à l'hypersensibilité peut s'expliquer par un renforcement négatif des comportements d'évitement qui souvent présentés par ces patients, renforce le lien entre symptômes et exposition. Des bénéfices secondaires peuvent également entretenir les symptômes ; une reconnaissance par certains groupes, le support familial, l'évitement de situations stressantes professionnelles, etc. (Mechanic, 1978). Enfin, la généralisation à d'autres sources de CEM, l'imprédictibilité des sources d'exposition et des symptômes ressentis peut conduire à une forme d'anxiété généralisée qui se traduit par une inquiétude profonde envers toute nouvelle forme de technologie et envers la société. Il n'est pas rare non plus qu'elle mène à des manifestations de type paranoïaque. L'évitement initial des sources de champs électriques et magnétiques incriminées tend à se généraliser à toute source de champs électriques et magnétiques et maintient ainsi le patient dans ses comportements d'évitement qui peuvent aller jusqu'à un isolement social important (en raison de la présence ubiquitaire de sources de champs électromagnétiques : lignes électriques, GSM, systèmes d'alarmes...) ainsi que des conditions de vie précaires (déménagement loin de toute source de champs électriques et magnétiques, réduction de l'utilisation d'éléctricité dans l'habitat au minimum) et/ou couteuses (aménagements d'une installation électrique avec réduction maximale de l'émission de champs électriques et magnétiques).

\section{Perspectives thérapeutiques}

Des études contrôlées à grande échelle sont nécessaires afin d'évaluer l'efficacité des différentes approches thérapeutiques. Étant donné l'incertitude relative aux agents susceptibles d'être responsables de l'HE, il est difficile de formuler un système d'intervention général (Bergqvist, 1995). Des examens médicaux et éventuellement dentaires doivent être pratiqués afin d'évacuer la possibilité d'une affection organique ou dentaire susceptible d'expliquer les plaintes exprimées. Dans environ 10 à $20 \%$ des cas, une maladie somatique a été identifiée qui explique les problèmes attribués à l'électricité (Hillert et Kolmodin-Hedman, 1997 ; Hillert et al., 2002b). Il est également important de faire un bilan des facteurs environnementaux, autres qu'électromagnétiques, susceptibles de contribuer aux symptômes observés.

Différentes approches ont été réalisées dont l'efficacité est variable d'un individu à l'autre.

L'acupuncture a également fait l'objet de recherche en relation avec le traitement de l'HE et s'est avérée bénéfique (Arnetz et al., 1995). Des traitements à base d'antioxydants (vitamine $\mathrm{C}$ et $\mathrm{E}$, Selenium) ont été testés, mais aucune différence significative n'a pu être mise en évidence entre pério- 
des de traitement placebo et par antioxydants au niveau des symptômes rapportés, de l'HE, du statut sérique oxydatif (Hillert et al., 2001b). Cette étude n'a pu confirmer l'hypothèse du stress oxydatif comme principal acteur de l'HE.

Certains programmes se fondent sur la réduction de l'exposition aux champs électriques et magnétiques, d'autres pas. La réduction de l'exposition présente des avantages et des inconvénients (Göthe, et al., 1995 ; Hillert, 1998; Hillert et al., 2002b). Elle est considérée comme efficace par les sujets hypersensibles interrogés de l'étude de Röösli et al., (2004), qu'il s'agisse de déconnecter l'électricité, de supprimer les sources intérieures, d'éviter les expositions ou de réaménager la maison voire de déménager. Cependant, il semble que la limitation des expositions peut présenter des effets limités voire néfastes dans certains cas, renforçant la conception de la relation cause-effet. Cette limitation peut accroître la peur et la charge de stress psychologique induite dans le groupe à risque et peut intensifier la symptomatologie (Göethe, et al., 1995). Certaines personnes expliquent la persistance des symptômes malgré les mesures de réduction de l'exposition par le fait qu'elles ont été exposées trop longtemps ou qu'elles sont exposées dans d'autres environnements (Hillert et Kolmodin-Hedman, 1997). De plus, l'évitement total de toute source de champs électromagnétiques étant pratiquement impossible, ces tentatives peuvent entraîner un stress chronique lié à l'incapacité de contrôler l'exposition (Hillert et al., 2002b). La faible consommation en médicaments et l'attrait pour une nourriture saine qui caractérisent les personnes qui se plaignent d'HE soulignent l'importance des phénomènes d'attribution envers l'environnement. Les hypersensibles attribuent généralement l'amélioration de leur bien-être à une réduction de l'exposition et réclament des modifications au niveau de l'environnement plutôt qu'au niveau d'ellesmêmes (Blomqvist, 1997).

En général et surtout dans les problèmes dermatologiques liés au travail sur écran, les programmes d'intervention se focalisent sur la réduction du stress et des situations organisationnelles néfastes et sur une intervention la plus précoce possible. De nombreuses adaptations sur les lieux de travail ont été réalisées qui portent sur l'environnement électromagnétique et les conditions de travail, avec plus ou moins de succès et sans pouvoir cerner avec précision quel facteur fut le plus efficace (Arnetz et Wiholm, 1997). Les facteurs psychosociaux professionnels sont cependant considérés comme le plus fort indicateur de risque de maintenance des symptômes dermatologiques dans les problèmes de dermatite de l'écran. Les mesures réalisées au niveau de l'environnement de travail, notamment celles destinées à réduire l'exposition à l'écran ou à d'autres appareils électriques ne sont pas associées à un meilleur pronostic (Stenberg et al., 2002). Stenberg rapporte également un moins bon pronostic pour les patients hypersensibles à l'électricité que les patients avec dermatite de l'écran. Ils présentent des symptômes plus nombreux et sont plus souvent dans l'incapacité de reprendre le travail, particulièrement les femmes. Ils fréquentent davantage psychiatres et psychologues dans leurs essais de traite- ment, à part égale avec les soins dentaires et les thérapies complémentaires. Cependant, le fait que ces patients aient eu souvent recours à de multiples traitements ne permet pas de tester leur efficacité individuelle. Des études de suivi sont donc nécessaires pour évaluer l'efficacité des divers traitements proposés.

Des publications tendent à démontrer l'efficacité des thérapies cognitivo-comportementales au niveau du handicap perçu et du degré d'hypersensibilité et soulignent l'efficacité d'une approche multidisciplinaire dans ce type de syndrome (Andersson et al., 1996 ; Bergdahl, 1995 ; Bergqvist et Vogel, 1997 ; Hillert et Kolmodin-Hedman, 1997 ; Hillert et al., 1998 ; Hillert et al., 1999 ; Hillert et al., 2002b). Comme le souligne le rapport de la commission européenne (Bergqvist et Vogel, 1997), il est important de prendre en considération les personnes hypersensibles à l'électricité et d'analyser leurs plaintes de manière adaptée, par une approche individuelle, adaptée à l'étape considérée (prévention, intervention, traitement) et sans focalisation sur un facteur unique. Dans certains cas, les tests de provocation permettent aux personnes hypersensibles de remettre en question la relation entre les symptômes de l'HE et l'exposition aux champs incriminés (Toomingas, 1996). Il s'agit donc d'un outil particulièrement utile qui doit être associé à un accompagnement psychologique dans les cas où l'expérience ne permet pas de confirmer l'HE.

\section{Conclusion}

Il n'existe pas de définition formelle ou de diagnostic d'HE en raison de la non-spécificité des symptômes rapportés, de l'apparente hétérogénéité des personnes affligées et de l'absence d'étiologie établie. Seule une définition opérationnelle existe à l'heure actuelle. L'HE est un syndrome essentiellement défini par le patient lui-même, lequel présente des plaintes qu'il attribue à l'usage ou la proximité d'appareils ou d'équipements émettant des champs électriques (CE), magnétiques (CM) ou électromagnétiques (CEM).

La plupart des études publiées, notamment les études de provocation, décrivent peu les patients qui se plaignent d'HE, leur histoire clinique, les circonstances d'apparition des symptômes, les autres expositions potentielles, les facteurs biologiques, psychologiques et psychosociaux, les caractéristiques symptomatiques. Il est important de mieux caractériser ce syndrome d'HE, car, actuellement, il n'est d'autre définition plus précise que celle qui se rapporte à la conviction des patients à propos de la cause de leur souffrance.

Les études sont encore trop peu nombreuses pour confirmer ou infirmer l'hypothèse de l'HE. Les études de provocation réalisées en double insu ne permettent pas de mettre en évidence une détection des champs électriques et magnétiques aux niveaux auxquels les personnes se plaignent par une perception directe des champs ou par l'apparition de leurs symptômes habituels. Le travail sur écran, pour reprendre le domaine actuellement le plus étudié en relation avec l'HE, 
est un travail qui implique également d'autres facteurs que les facteurs électriques, susceptibles d'aggraver les symptômes dermatologiques tout comme les symptômes fonctionnels de type céphalée, fatigue etc. L'attribution causale de la réaction à « l'électricité » reste une hypothèse qui doit être encore testée dans les études futures. Il est en effet possible que des facteurs physiques, en l'occurrence l'exposition aux champs électromagnétiques puissent interagir avec des facteurs psychologiques et la susceptibilité individuelle à développer un profil clinique déterminé. Les patients qui se plaignent d'HE forment un groupe très hétérogène et il est possible que plusieurs mécanismes explicatifs interviennent, en fonction des situations et des personnes. Il n'est pas impossible que des modèles de croyance, une vulnérabilité psychologique particulière ou des facteurs de stress puissent engendrer des groupes de personnes plus sensibles à certains facteurs environnementaux dont l'électricité. Hillert et al. soulignent en effet la présence de situations de stress accru ou d'événements de vie négatifs avant l'apparition des symptômes (Hillert, 2002b).

L'origine de l'HE serait davantage multifactorielle, ce qui incite à la recherche de facteurs internes et externes, individuels et environnementaux, dans une perspective biopsychosociale. L'étiologie multifactorielle de ce syndrome souligne l'importance d'une approche globale et multidisciplinaire afin de couvrir au mieux les différents aspects impliqués dans la symptomatologie. Étant donné les conséquences que peut entraîner ce syndrome sur le plan individuel, en terme d'isolement social et professionnel, en termes de consommation de soins de santé, il est important de prendre en considération la souffrance exprimée par ces personnes le plus rapidement possible, avant l'aggravation de la symptomatologie. La souffrance de ces personnes est bien réelle, elle nécessite des actions de prévention, d'intervention précoce et de traitement.

Les recherches futures devraient permettre d'améliorer la définition de l'HE, de fournir des critères diagnostiques clairement définis et de mieux appréhender la ou les étiologie(s) de ce syndrome. Il convient également de réaliser une communication adéquate du risque lié à l'exposition aux champs électromagnétiques. Cette communication doit rejoindre les préoccupations et les craintes du public afin de prévenir l'apparition ou la cristallisation des premiers symptômes. Ajoutons pour terminer que l'étude de l'HE n'exclut pas la possibilité d'effets psychologiques (sur les fonctions cognitives ou l'humeur) ou biologiques (au niveau du cancer, des maladies dégénératives...) dans la population générale, liés à l'exposition aux champs électriques, magnétiques et/ou électromagnétiques. Il s'agit dans ce cas d'études d'un type différent qui s'inscrit dans le cadre de la recherche des effets sur la santé de l'exposition à des champs électriques, magnétiques et électromagnétique sans référence a priori à un groupe d'individus qui se plaint d'être plus sensible que les autres personnes à cette exposition. De nombreuses recherches ont été réalisées ou sont toujours en cours dans ce domaine.

\section{Références}

Al-Khlaiwi, T., Meo, S.A., 2004. Association of mobile phone radiation with fatigue, headache, dizziness, tension and sleep disturbance in Saudi population. Saudi Medical Journal 25 (6), 732-736.

Andersson, B., Berg, M., Arnetz, B.B., Melin, L., Langlet, I., Liden, S., 1996. A cognitive-behavioral treatment of patient suffering from "electric hypersensitivity". Subjective effects and reactions in a double-blind provocation study. Journal of Occupational and Environmental Medicine 38 (8), 752-758.

Antilla, K., 2000. Mycotoxins, fungus and 'electrohypersensitivity'. Medical Hypotheses 55 (3), 208-214.

Arnetz, B.B., Berg, M., Anderzén, I., Lundeberg, T., Haker, E., 1995. A nonconventional approach to the treatment of Environmental Illness. J. Occup. Environ. Med. 37, 838-844.

Arnetz, B.B., Berg, M., 1996. Melatonin and adrenocorticotropic hormone levels in video display unit workers during work and leisure. Journal of Occupational Environmental Medicine 38 (11), 1108-1110.

Arnetz, B.B., Wiholm, C., 1997. Technological stress: psychophysiological symptoms in modern offices. Journal of Psychosomatic Research 43 (1), $35-42$.

Arnetz, B.B., 1998. Environmental Illness: Multiple Chemical Sensitivity, Sick Building Syndrome, Electric and Magnetic Field Disease. In: Lundberg, A. (Ed.), The environment and mental health. A guide for clinicians. Lawrence Erlbaum Associates, London, pp. 115-146.

Bailer, J., Rist, F., Rudolf, A., Staehle, H.J., Eickholz, P., Triebig, G., Bader, M., Pfeifer, U., 2001. Adverse health effects related to mercury exposure from dental amalgam fillings: toxicological or psychological causes? Psychological Medicine 31 (2), 255-263.

Barsky, A.J., Klerman, G.L., 1983. Overview: hypochondriasis, bodily complaints, and somatic styles. American Journal of Psychiatry 140 (3), 273-283.

Berg, M., 1988. Skin problems in workers using visual display terminals. A study of 201 patients. Contact Dermatitis 19 (5), 335-341.

Berg, M., Hedblad, M.A., Erhard, K., 1990a. Facial skin complaints and work at visual display units: a histopathological study. In: Acta Dermatovenereologica. pp. 216-220.

Berg, M., Liden, S., Axelson, O., 1990b. Skin complaints and work at visual display units: an epidemiological study of office employees. J. Am. Acad. Dermatol. 22, 621-625.

Berg, M., Arnetz, B., Liden, S., Eneroth, P., Kallner, A., 1992. Technostress, a psychophysiological study of employees with VDU associated skin complaints. Journal of Occupational Medicine 34 (7), 698-701.

Bergdahl, J., 1995. Psychologic aspects of patients with symptoms presumed to be caused by electricity or visual display units. Acta Odontologica Scandavica 53, 304-310.

Bergdahl, J., Anneroth, G., Stenman, E., 1994. Description of people with symptoms presumed to be caused by electricity or visual display units oral aspects. Scand. J. Dent. Res. 102, 41-45.

Bergdahl, J., Bergdahl, M., 2001. Environmental illness: evaluation of salivary flow, symptoms, diseases, medications, and psychological factors. Acta Odontologica Scandinavica 59 (2), 104-110.

Bergdahl, J., Tillberg, A., Stenman, E., 1998. Odontologic survey of referred patients with symptoms allegedly caused by electricity or visual display units. Acta Odontol. Scand. 56, 303-307.

Bergqvist, U., 1995. Hypersensitivity to electricity-a round table discussion. In: Grieco, A., Molteni, B., Piccoli, B., Occhipinti, E. (Eds.), Work with display Units. Elsevier Science.

Bergqvist, U., Wahlberg, J.E., 1994. Skin symptoms and disease during work with visual display terminals. Contact Dermatitis 30 (4), 197-204.

Possible health implication of subjective symptoms and electromagnetic fields. In: Bergqvist, U., Vogel, E. (Eds.), 1997, A report by a European group of experts for the European Commission, DG V. National Institute for Working Life, Solna, Sweden (Arbeteoch Hälsa 1997: 19).

Blomqvist, A.C., 1997. "Hypersensitivity to electricity" in the office; symptoms and improvement. International Journal of Occupational Safety and Ergonomics 3 (3-4), 129-140. 
Chia, S.E., Chia, H.P., Tan, J.S., 2000. Prevalence of headache among handheld cellular telephone users in Singapore: a community study. Environ. Health Perspect. 108, 1059-1062.

Cox, R.A., Luxton, L.M., 2000. Cerebral symptoms from mobile telephones (letter). Occup. Environ. Med. 57, 431.

Crasson, M., 2003. 50-60 Hz electric and magnetic field effects on cognitive function in humans: a review. Radiation Protection Dosimetry 106 (4), 333-340.

D’Andrea, J.A., Adair, E.R., De Lorge, J.O., 2003. Behavioral and cognitive effects of microwave exposure. Bioelectromagnetics (Supplement 6), S39-S62.

Eriksson, N., Höög, J., Sandström, M., Stenberg, B., 1997a. Facial skin symptoms in office workers. A five-year follow-up study. J. Occup. Environ. Med. 39, 108-118.

Eriksson, N., Höög, J., Hansson Mild, K., Sandström, M., Stenberg, B., 1997b. The psychological work environment and skin symptoms among visual display terminal workers: a case referent study. Int. J. Epidemiol. 26, 1250-1257.

Escobar, J.I., Hoyos-Nervi, C., Gara, M., 2002. Medically unexplained physical symptoms in medical practice: a psychiatric perspective. Environ. Health Perspect. 110 (Suppl 4), 631-636.

Flodin, U., Seneby, A., Tegenfeldt, C., 2000. Provocation of electric hypersensitivity under everyday conditions. Scand. J. Work Environ. Health 26, 93-98

Frick, U., Rehm, J., Eichhammer, P., 2002. Risk perception, somatization, and self report of complaints related to electromagnetic fields--a randomized survey study. International Journal of Hygiene and Environtal Health 205 (5), 353-360.

Gangi, S., Johansson, O., 1997. Skin changes in "screen dermatitis" versus classical UV and ionizing irradiation-related damage-similarities and differences. Exp. Dermatol. 6, 283-291.

Gangi, S., Johansson, O., 2000. A theoretical model based upon mast cells and histamine to explain the recently proclaimed sensitivity to electric and/or magnetic fields in humans. Medical Hypotheses 54 (4), 663-671.

Göthe, C.J., Molin, C., Nilsson, C.G., 1995. The environmental somatization syndrome. Psychosomatics 36 (1), 1-11.

Hamnerius, Y., Sjöberg, P., 1994. Investigation of provoked hypersensitivity reactions from the electric and magnetic fields of a video display unit. In: Simunic, D. (Ed.), Proceedings of the COST 244 meeting on Electromagnetic Hypersensitivity. Graz, Austria, pp. 44-45 COST244.

Hamnerius, Y., Agrup, G., Galt, S., Nilsson, R., Sandblom, J., Lindgren, R., 1994. Double blind provocation study of reactions associated with exposure to electromagnetic fields from VDUs. In: Simunic, D. (Ed.), Proceedings of the COST 244 meeting on Electromagnetic Hypersensitivity. Graz, Austria, pp. 41-43 COST244.

Hietanen, M., Hämäläinen, A.M., Husman, T., 2002. Hypersensitivity symptoms associated with exposure to cellular telephones: no causal link. Bioelectromagnetics 23, 264-270.

Hillert, L., Kolmodin-Hedman, B., 1997. Hypersensitivity to electricity: sense or sensibility? Journal of Psychosomatic Research 42 (5), 427432.

Hillert, L., 1998. Hypersensitivity to electricity: management and intervention programs. In: Leitgeb, N. (Ed.), Proceedings of the COST 244bis international workshop on Electromagnetic fields and Non-Specific Health Symptoms. Graz, Austria, pp. 17-30 COST244bis.

Hillert, L., Kolmodin-Hedman, B., Dölling, B.F., Arnetz, B.B., 1998. Cognitive behavioural therapy for patients with electric sensitivity-a multidisciplinary approach in a controlled study. Psychother. Psychosom. 67, 302-310.

Hillert, L., Hedman, B.K., Soderman, E., Arnetz, B.B., 1999. Hypersensitivity to electricity: working definition and additional characterization of the syndrome. Journal of Psychosomatic Research 47 (5), 429-438.

Hillert, L., (2001). Hypersensitivity to electricity: Symptoms, risk factors and therapeutic interventions. PhD thesis. ISBN: 91-7349-016-4. In Karolinska Institut, Stockholm, Sweden. Site de 1'Institut Karolinska : http://diss.kib.ki.se/2001/91-7349-016-4/ (page consultée le 3 septembre 2004).
Hillert, L., Flato, S., Georgellis, A., Arnetz, B.B., Kolmodin-Hedman, B., 2001a. Environmental illness: fatigue and cholinesterase activity in patients reporting hypersensitivity to electricity. Environmental Research 85 (3), 200-206.

Hillert, L., Kolmodin-Hedman, B., Eneroth, P., Arnetz, B.B., 2001b. The effect of supplementary antioxidant therapy in patients who report hypersensitivity to electricity: a randomized controlled trial. Medscape General Medicine 3 (2), 11.

Hillert, L., Berglind, N., Arnetz, B.B., Bellander, T., 2002a. Prevalence of self-reported hypersensitivity to electric or magnetic fields in a population-based questionnaire survey. Scandinavian Journal of Work Environment and Health 28 (1), 33-41.

Hillert, L., Savlin, P., Berg, A., Heidenberg, A., Kolmodin-Hedman, B., 2002b. Environmental illness-effectiveness of a salutogenic groupintervention programme. Scandinavian Journal of public health 30 (3), $166-175$.

Hocking, B., 1998. Preliminary report: symptoms associated with mobile phone use. Occupational Medicine (Oxford) 48, 357-360.

International Agency for Research on Cancer (IARC), 2002. IARC Monographs on the evaluation of carcinogenic risk to humans, Vol. 80, Nonionizing radiation, part 1: static and extremely low-frequency (ELF) electric and magnetic fields. IARCPress, Lyon, France.

International Programme on Chemical Safety/World Health Organization (IPCS/WHO), 1996. Conclusions and recommendations of a workshop on multiple chemical sensitivities (MCS). Regulatory Toxicology Pharmacology 24, S188-S189.

Johansson, O., Hilliges, M., Björnhagen, V., Hall, K., 1994. Skin changes in patients claiming to suffer from screen dermatitis: a two-case open-field provocation study. Exp. Dermatol. 3, 234-238.

Jorulf, L., Barregard, L., Hagberg, S., Krogstad, A.L., Ran, A., 1993. A longitudinal study of symptoms in office VDU-work at different room temperatures. A preliminary report. In: Luczak, H., Cakir, A., Cakir, G. (Eds.), Work with Display Units'92. Selected proceedings of the 3rd International Conference WWDU '92, Berlin, Germany. Elsevier Science, Amsterdam, pp. 54-58.

Kipen, H.M., Fiedler, N., 2002. The role of environmental factors in medically unexplained symptoms and related syndromes: conference summary and recommendations. Environ. Health Perspect. 110 (Suppl 4), 591-595.

Knave, B., Bergqvist, U., Wibom, R., 1992. " Hypersensitivity to electricity "- a workplace phenomenon related to low frequency electric and magnetic fields. the proceedings of the Eighth International Congress of the International Radiation Protection Association, Worldwide achievement in public and occupational health protection against radiation, vol. II, (1121-1124), Montreal, Canada.

Koivisto, M., Haarala, C., Krause, C.M., Revonsuo, A., Laine, M., Hämäläinen, H., 2001. GSM phone signal does not produce subjective symptoms. Bioelectromagnetics 22, 212-215.

Kunsch, B., 1994. Electromagnetic fields and risk perception. In: Simunic, D. (Ed.), Proceedings of the COST 244 meeting on Electromagnetic Hypersensitivity. Graz, Austria, pp. 58-67 COST244.

Leitgeb, N., 1998. Electromagnetic hypersensitivity. In: Leitgeb, N. (Ed.), Proceedings of the COST 244bis international workshop on Electromagnetic fields and Non-Specific Health Symptoms. Graz, Austria, pp. 8-13 COST244bis.

Leitgeb, N., Schrötter, J., 2003. Electrosensibility and electromagnetic hypersensitivity. Bioelectromagnetics 24, 387-394.

Levallois, P., Neutra, R., Lee, G., Hristova, L., 2002. Study of self-reported hypersensitivity to electromagnetic fields in California. Environ. Health Perspect. 110 (Suppl 4), 619-623.

Levallois, P., 2002. Hypersensitivity of human subjects to environmental electric and magnetic field exposure: a review of the literature. Environmental health perspectives 110 (4), 613-618.

Liden, C., Wahlberg, J.E., 1985. Work with video display terminals among office employees. V. Dermatologic factors. Scandinavian Journal of Work Environment and Health 11 (6), 489-493. 
Liden, S., 1996. "Sensitivity to electricity"-a new environmental epidemic. Allergy 51 (8), 519-524.

Lonne-Rahm, S., Andersson, B., Melin, L., Schultzberg, M., Arnetz, B., Berg, M., 2000. Provocation with stress and electricity of patients with "sensitivity to electricity". Journal of Occupational and Environmental Medicine 42 (5), 512-516.

Lyskov, E., Sandström, M., Hansson Mild, K., 2001. Provocation study of persons with perceived electrical hypersensitivity and controls using magnetic field exposure and recording of electrophysiological characteristics. Bioelectromagnetics 22 (7), 457-462.

MacGregor, D.G., Fleming, R., 1996. Risk perception and symptom reporting. Risk Analysis 16 (6), 773-783.

McMahan, S., Meyer, J., 1995. Symptom prevalence and worry about high voltage transmission lines. Environmental Research 70 (2), 114-118.

Mechanic, D., 1978. Effects of psychological distress on perceptions of physical health and use of medical and psychiatric facilities. Journal of Human Stress 4 (4), 26-32.

Müeller, C.H., Krueger, H., Schierz, C., 2002. Project NEMESIS: perception of a $50 \mathrm{~Hz}$ electric and magnetic field at low intensities (laboratory experiment). Bioelectromagnetics 23 (1), 26-36.

Oftedal, G., Wilén, J., Sandström, M., Hansson Mild, K.H., 2000. Symptoms experienced in connection with mobile phone use. Occup. Med. 50, 237-245.

Oftedal, G., Nyvang, A., Moen, B.E., 1999. Long-term effects on symptoms by reducing electric fields from visual display units. Scandinavian Journal of Work Environment and Health 25 (5), 415-421.

Oftedal, G., Vistnes, A.I., Rygge, K., 1995. Skin symptoms after the reduction of electric fields from visual display units. Scandinavian Journal of Work Environment and Health 21 (5), 335-344.

OMS, Organisation mondiale de la santé, Aide-mémoire ${ }^{\circ} 184$, vérifié en mai 1998. Site de l'OMS, http://www.who.int/docstore/peh-emf/ publications/facts_press/ffact/ffs 184 .html (page consultée le 3 septembre 2004).

Örtendahl, J., Högstedt, P., Holland, R.L., 1991. Mercury vapor release from dental amalgam in vitro caused by magnetic fields generated by CRT's and electrical cutting procedures. Swed. Dent. J. 15, 31.

Pennebaker, J.W., 1994. Psychological bases of symptom reporting: perceptual and emotional aspects of chemical sensitivity. Toxicology and Industrial Health 10 (4/5), 497-511.

Rea, W.J., Pan, Y., Fenyves, E.J., Sujisawa, I., Suyama, H., Samadi, N., Ross, G.H., 1991. Electromagnetic field sensitivity. Journal of Bioelectricity 10 (1 and 2), 241-256.

Röösli, M., Moser, M., Baldinini, Y., Meier, M., Braun-Fahrlander, C., 2004. Symptoms of ill health ascribed to electromagnetic field exposure-a questionnaire survey. International Journal of Hygiene and Environmental Health 207 (2), 141-150.

Sandström, M., Hansson Mild, K., Stenberg, B., Wall, S., 1995. Skin symptoms among VDT workers and electromagnetic fields. A case-referent study. Indoor Air 5, 29-37.

Sandström, M., Lyskov, E., Berglund, A., Medvedev, S., Hansson Mild, K., 1997. Neurophysiological effects of flickering light in patients with perceived electrical hypersensitivity. Journal of Occupational Environmental Medicine 39 (1), 15-22.

Sandström, M., Wilén, J., Oftedal, G., Hansson Mild, K., 2001. Mobile phone use and subjective symptoms. Comparison of symptoms experienced by users of analogue and digital mobile phones. Occup. Med. 51, $25-35$.

Santini, R., Seigne, M., Bonhomme-Faivre, L., Bouffet, S., Defrasne, E., Sage, M., 2001a. Symptômes rapportés par les utilisateurs de téléphones mobiles cellulaires. Pathol. Biol. (Paris) 49, 222-226.

Santini, R., Santini, P., Seigne, M., Danze, J.M., 2001b. Symptômes exprimés par des riverains de stations relais de téléphonie mobile. Presse Med. 32, 1594.
Silny, J., 1999. Electrical hypersensitivity in humans-fact or fiction? Zentralbl Hyg Umweltmedizin 202 (2-4), 219-233.

Skulberg, K.R., Skyberg, K., Eduard, W., Goffeng, L.O., Vistnes, A.I., Levy, F., et al., 2001. Effects of electric field reduction in visual display units on skin symptoms. Scandinavian Journal of Work Environment and Health 27 (2), 140-145.

Sparks, P.J., 2000a. Idiopathic environmental intolerances: overview. Occupational Medicine 15 (3), 497-510.

Sparks, P.J., 2000b. Diagnostic evaluation and treatment of the patient presenting with idiopathic environmental ntolerance. Occupational Medicine 15 (3), 601-609.

Staudenmayer, H., 2000. Psychological treatment of psychogenic idiopathic environmental intolerance. Occupational Medicine 15 (3), 627-646.

Stenberg, B., Eriksson, N., Hansson Mild, K., Höög, J., Sandström, M., Sundell, J., et al., 1995. Facial skin symptoms in visual display terminal (VDT) workers. A case-referent study on personal, psychosocial, building and VDT related risk indicators. Int. J. Epidemiol. 24, 796-803.

Stenberg, B., Bergdahl, J., Edvardsson, B., Eriksson, N., Linden, G., Widman, L., 2002. Medical and social prognosis for patients with perceived hypersensitivity to electricity and skin symptoms related to the use of visual display terminals. Scandinavian Journal of Work Environment and Health 28 (5), 349-357.

Stevens, R.G., Davis, S., 1996. The melatonin hypothesis: electric power and breast cancer. Environ. Health Perspect. 104 (Suppl 1), 135-140.

Swanbeck, G., Bleeker, T., 1989. Skin problems from visual display units. Acta Derm. Venereol. 69, 46-51.

Swanborn, A., Vossen, S., Van Leersum, B., Ouwens, M., Mäkel, W., 2003. Effects of global communication system radio-frequency fields on wellbeing and cognitive functions of human subjects with and without subjective complaints. (TNO FEL-03-C148 report. TNO, Physics and Electronics Laboratory, The Netherlands.

Toomingas, A., 1996. Provocation of the electromagnetic distress syndrome. Scandinavian Journal of Work Environment and Health 22 (6), 457-458.

Trimmel, M., Schweiger, E., 1998. Effects of an ELF $(50 \mathrm{~Hz}, 1 \mathrm{mT})$ electromagnetic field (EMF) on concentration in visual attention, perception and memory including effects of EMF sensitivity. Toxicol. Lett. 96 (97), 377-382.

Wadman, C., Bergqvist, U., Medhage, Ö., Linder, G., Knave, B., 1996. In: Elöverkänslighet - en studie bland kontoersarbetare. (Hypersensitivity to electricity - a study among office workers. In Swedish.). National Institute for Working Life, Solna, Sweden (Arbetslivsrapport 1996:5).

Wang, T., Hawkins, L.H., Rea, W.J., 1994. Effects of ELF magnetic fields on patients with chemical sensitivities. In: Simunic, D. (Ed.), Proceedings of the COST 244 meeting on Electromagnetic Hypersensitivity. Graz, Austria, pp. 123-132 COST244.

Wartenberg, D., Greenberg, M., 1992. Methodological problems in investigating disease clusters. Science of the Total Environment 127 (1-2), 173-185.

Wennberg, A., Franzen, O., Paulsson, L.E., 1994. Electromagnetic provocation of subjects with "Electric hypersensitivity". In: Simunic, D. (Ed.), Proceedings of the COST 244 meeting on Electromagnetic Hypersensitivity. Graz, Austria, pp. 133-139 COST244.

Wessely, S., Nimnuan, C., Sharpe, M., 1999. Functional somatic syndromes: one or many? Lancet 354, 936-939.

Wibom, R., Nylén, P., Wennberg, A., Flimmer, F.L., 1995. Flicker from fluorescent tubes. A possible contributary cause of hypersensitivity to electricity. National Institute for Working Life, Solna, Sweden (Undersökningsrapport 1995:31).

Wilen, J., Sandström, M., Hansson Mild, K., 2003. Subjective symptoms among mobile phone users-a consequence of absorption of radiofrequency fields? Bioelectromagnetics 24, 152-159.

Winters, W., Devriese, S., Van Diest, S., Nemery, B., Veulemans, H., Eelen, P., et al., 2003. Media warnings about environmental pollution facilitate the acquisition of symptoms in response to chemical substances. Psychosom. Med. 65, 332-338. 